\title{
Global laminate optimization on geometrically partitioned shell structures
}

\section{Journal Article}

Author(s):

Keller, David

Publication date:

2010

Permanent link:

https://doi.org/10.3929/ethz-b-000158279

Rights / license:

In Copyright - Non-Commercial Use Permitted

Originally published in:

Structural and Multidisciplinary Optimization 43(3), https://doi.org/10.1007/s00158-010-0576-9 


\title{
Global laminate optimization on geometrically partitioned shell structures
}

\author{
David Keller
}

Received: 24 February 2010 / Revised: 15 August 2010 / Accepted: 21 September 2010 / Published online: 20 October 2010

(C) Springer-Verlag 2010

\begin{abstract}
A method aimed at the optimization of locally varying laminates is investigated. The structure is partitioned into geometrical sections. These sections are covered by global plies. A variable-length representation scheme for an evolutionary algorithm is developed. This scheme encodes the number of global plies, their thickness, material, and orientation. A set of genetic variation operators tailored to this particular representation is introduced. Sensitivity information assists the genetic search in the placement of reinforcements and optimization of ply angles. The method is investigated on two benchmark applications. There it is able to find significant improvements. A case study of an airplane's side rudder illustrates the applicability of the method to typical engineering problems.
\end{abstract}

Keywords Laminate optimization - Evolutionary algorithm $\cdot$ Sensitivity analysis

\section{Introduction}

The sensitivity of the mechanical properties of laminated structures to the choice of material systems employed, the spatial distribution of different materials within the structure, and the local orientation of fiber reinforcements in the laminate offers for an application specific tuning.

D. Keller $(\bowtie)$

Centre of Structure Technologies, ETH Zürich,

8092 Zürich, Switzerland

e-mail: davidkeller@alumni.ethz.ch
However, due to the large number of variables optimal designs may be hidden from human intuition. Hence, numerical optimization of laminated composites is a field of active research.

A key to the numerical optimization of laminated structures lies in the parameterization of the laminate stack. The most simple setup starts from existing designs and takes ply angles as continuous decision variables. Analytical approaches to the optimization of ply angles (like e.g., Prager 1970; Pedersen 1989, 1991; Duvaut et al. 2000; Khosravi and Sedaghati 2008; Vannucci et al. 2009) are only available for geometrically simple cases. The application of mathematical programming either risks to get stuck in a local optimal solution (which may be accepted in some situations, like e.g., Topal and Uzman 2008; Johansen et al. 2009) or requires extensive tuning of the optimizer itself in order to converge to the global optimal solution (e.g., Moita et al. 2000; Bruyneel and Fleury 2002). So far, a transformation of the original to a convex optimization problem e.g., by lamination parameters is not available for general shell structures but only for simple geometries (Grenestedt 1990, 1991; Hammer et al. 1997; Miki 1982; Foldager et al. 1998; Abdalla et al. 2007). Furthermore, the back-transformation from optimal lamination parameters to a real lay-up is a non-trivial task and may require a second optimization step (cf. Autio 2000; Herencia et al. 2007). Although numerically expensive, evolutionary approaches have been applied to ply angle optimization (e.g., Gürdal et al. 1999; Walker and Smith 2003; Naik et al. 2008). In order to reduce the computational requirements of a global search by evolutionary algorithms (EAs) an iterative procedure including a local gradient-based search is proposed by Huang and Haftka (2005).

Combinations of ply angle optimization with the optimization of ply materials (cf. e.g., Le Riche and Haftka 
1995; Le Riche et al. 1995) or the optimization of ply thicknesses (like Fukunaga and Vanderplaats 1991; Omkar et al. 2008) lead to problems with partially discrete search space. Stochastic optimizers like EAs are employed there (Adali et al. 2003; Lopez et al. 2009; Naik et al. 2008; Akbulut and Sonmez 2008).

Whilst for in-plane loadings the laminate stiffness and strength depends only on the fiber orientations, thicknesses, and elastic properties of the single laminae, out-of-plane (e.g., bending, buckling, or frequency) situations involve the position of each lamina in the laminate stack as a further decision variable.

Apart from mathematical programming (e.g., Tsau and Liu 1995; Farshi and Rabiei 2007), stochastic optimizers have repeatedly and successfully been applied to the optimization of the laminate stack: These include variants of EAs (Kogiso et al. 1994; Soremekun 1997; Todoroki and Haftka 1997, 1998; Liu et al. 2000; Todoroki and Ishikawa 2004; Rao and Arvind 2005; Pelletier and Vel 2006; Cho and Rowlands 2007; Kim 2007; Park et al. 2008; Pelegri and Kedlaya 2008; Beluch 2007), heuristic branch-and-bound methods (Matsuzaki and Todoroki 2007; Todoroki and Sekishiro 2007), tabu-search (Pai et al. 2003), particle swarm optimization (Suresh et al. 2007; Omkar et al. 2008), or ant-colony optimization (Aymerich and Serra 2008).

A class of optimization methods for locally varying laminate properties are pioneered by Zowe et al. (1997), Kočvara and Zowe (1998) and Ben-Tal et al. (1999) under the name free material approach (FMA). There, the elastic properties at any point in the design space are decision variables. The method generates an optimal material distribution in terms of virtual homogenized material properties by the means of a direct search method and finite elements. In order to map the virtual elastic properties back to a real and manufacturable lay-up, some potentially expensive post-processing is required. A method to map the results of FMA to a tape-laying lamination plan is proposed by Hörnlein et al. (2001).

Voxel-based parameterization schemes covering the structure with a predefined set of virtual plies, each associated with an existing real material, a thickness and ply angle, and then searching for sets of voxels where these virtual plies should become existent or vanish have been proposed several times. The discrete material optimization (DMO) approach has been pioneered by Lund and Stegmann (2005, 2006), Stegmann (2005) and Stegmann and Lund (2005).

A group of methods manually partitions the structure into geometric regions (or sections) in a first step. Then, in a second step a laminate parameterization scheme can be applied to the sections independently. In order to ensure the global cohesion of the structure, some plies covering and hence connecting several sections have to be applied (Adams et al. 2004; Seresta et al. 2007). Furthermore, the optimization of the thickness of each section requires special attention: Whilst some methods fall back on (quasi-) isotropic models to determine thickness distributions (like Rastogi 2004; Roche 2004), Giger and Ermanni (2005) encode the physical existence of plies by additional decision variables.

The genetic encoding of a locally variable laminate consisting of global patches is developed. Prior investigations require a high number of structural evaluations (Giger et al. 2008) or show a considerable spread in the optimization results of different optimization runs (Giger 2007, Chapter 7). This study aims at investigating possibilities to embed gradient information in a genetic encoding in order to improve the solution quality and the rate of convergence of the method.

\section{Method}

\subsection{An asynchronous, parallel evolutionary algorithm scheme}

EAs are a class of biologically inspired, population-based global search algorithms. One can discern four different branches in evolutionary computation: evolutionary programming, genetic algorithms, evolution strategies, and genetic programming. In this study, the more modern term EA coined by Bentley (1999) is preferred.

The investigated parallel algorithm is outlined in Algorithm 1. A flowchart of its state is depicted in Fig. 1. The main concepts are borrowed from König (2004) and Wintermantel (2004). The state of the algorithm is characterized by a set of candidate solutions, i.e., a population of individuals. Each individual is equipped with a fitness, grading how well it complies to the structural demands formulated in the optimization problem. Based on the fitness, mating selection (State 4) draws samples-the so-called genitorswhich are allowed for reproduction. Variation (State 5) then either recombines the genetic information of several genitor individuals (crossover) or induces random perturbations (mutation) in order to generate offspring. Evaluation (State 6) computes the fitness of new offspring. Environmental selection (State 7) decides which individuals of the former parent population and the new offspring build the new parents. The term replacement is sometimes used as a synonym for environmental selection, although it only applies to one particular selection scheme where the offspring replaces the parents. Hence, the more general term environmental selection shall be preferred. The proposed scheme executes mating selection, variation, environmental selection, and evaluation in parallel. A fixed-size population evolved over subsequent generations is replaced by a pool 


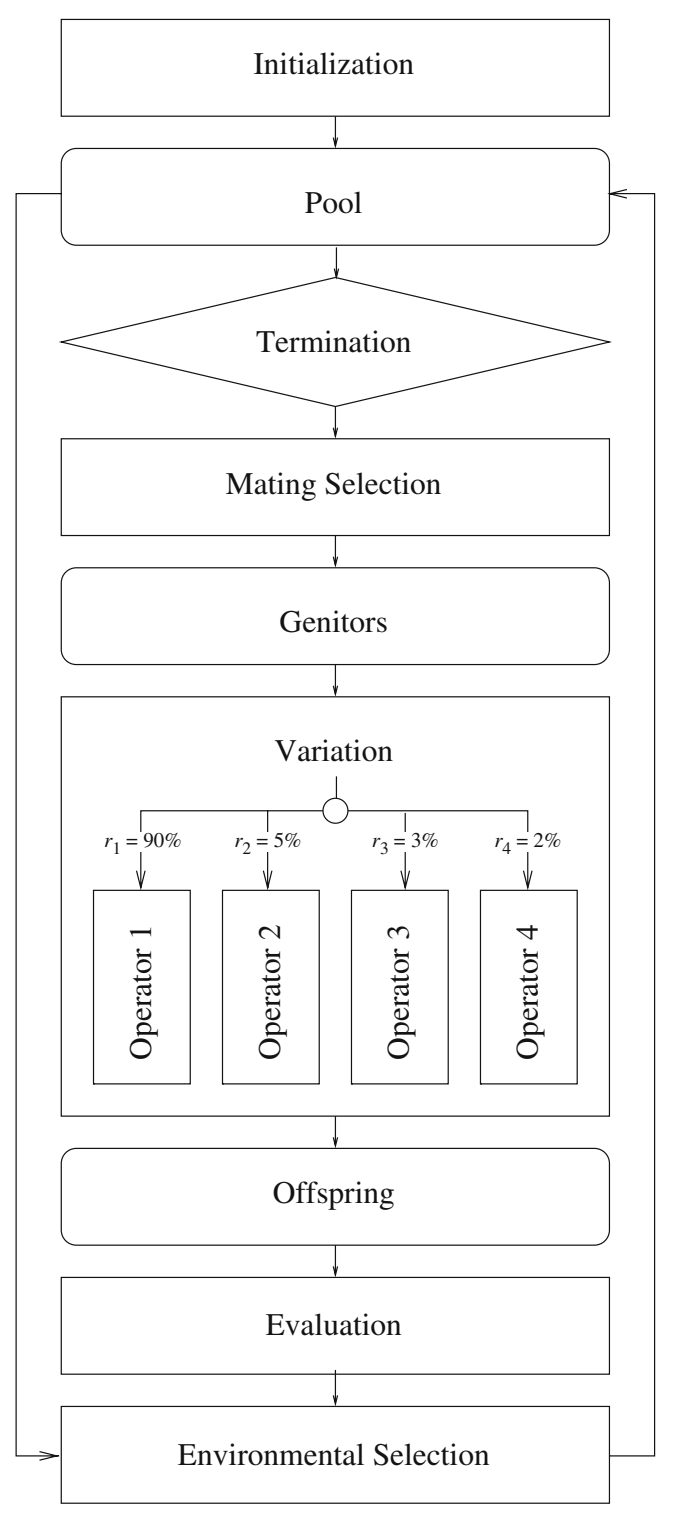

Fig. 1 Flowchart of evolutionary algorithm scheme (Algorithm 1)

$M$. The pool allows for continuous evaluations. The evaluation state includes computationally expensive simulations. Hence a parallelization of this state leads to a significant speedup of the optimization run (cf. Cantu-Paz 1997). Variation operations embedding iterative local searches (as detailed in the following) have considerably larger runtimes than stochastic operations. Thus, asynchronous (i.e., nonblocking) parallelization of the variation state is required to fully exploit the system capacity. Asynchronous parallelization requires adaptions in the environmental selection state. An age-based mechanism is employed to shift selection operations from the population to the individual level. An in-depth discussion of the parallel implementation is outside the scope of this study but can be found in Keller (2010a, b). The following sections focus on the representation concept, i.e., the structure of the information stored in a candidate solution $\mathbf{x}$. An overview of problem specific representation concepts in evolutionary structural optimization is given in e.g., Kicinger et al. (2005) and Giger (2007). A representation concept includes two aspects: a description on how to encode attributes of candidate solutions and a set of variation operations modifying these encoded attributes. Thus, the variation state needs special consideration when designing new genetic encodings. The implementation of the variation state in the investigated algorithm is based on a proportional configuration already employed in König (2004), Wintermantel (2004), Giger (2007), Zehnder (2008) and Sauter (2008): Each single variation operator is equipped with a user-defined application rate $r_{i}$. In each execution thread of the parallel implementation, only one variation operation gets applied by chance. Hence, mating selection is executed in order to identify the number of genitors required as parent individuals for the particular choice of a variation operator. This is also illustrated in Fig. 1 with an exemplary setup for the rates $r_{i}$.

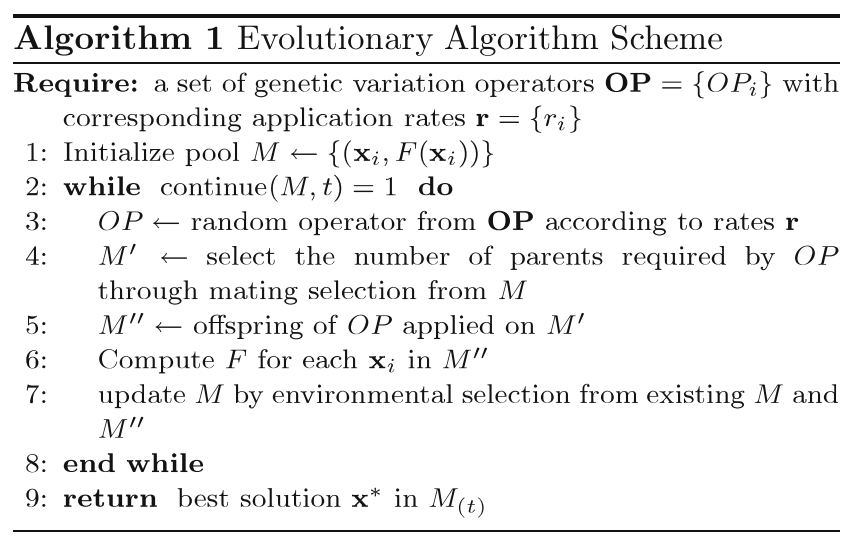

\subsection{Building laminates from global plies}

If locally varying laminates shall be used within a structure, the global cohesion has to be assured. The patch concept developed by Zehnder and Ermanni (2006) and Zehnder (2008) enables for this by parameterizing global plies, i.e., patches. The position, size, shape, material, and orientation of the patches may be optimized. Due to their overlappings, patches form sections on the structure with different laminates (Fig. 2). An extension of the method is proposed in Giger (2007, Chapter 5) where a variable-length genotype in an evolutionary algorithm enables to optimize the number of patches as well. A finite element-wise approach to parameterize the shape of reinforcement patches is investigated in Giger et al. (2008). An abstraction of this method to operate on predefined geometrical zones instead of finite elements can be found in Kaufmann (2006) (revisited in Chapter 7 of Giger 2007). 


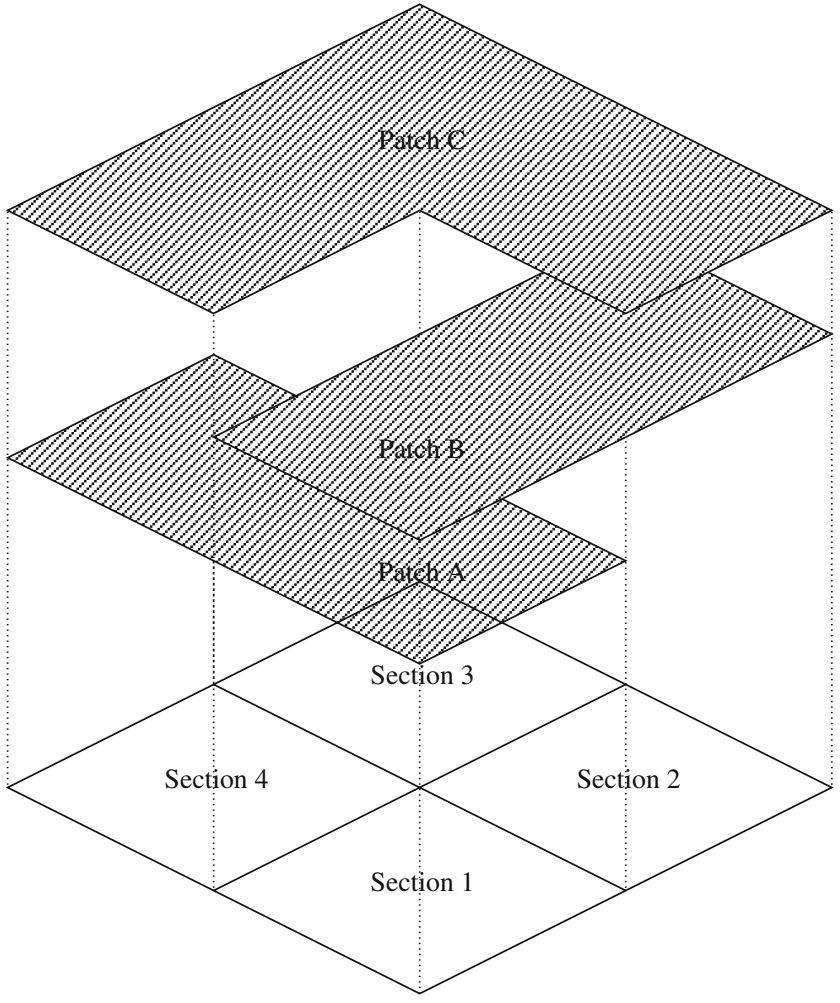

Fig. 2 Mapping of three patches (A, B, and C) to four sections (1, 2, 3, and 4). Section 1 consist of patches A and B, Section 2 of B and C, Section 3 of only C, and Section 4 is a laminate from A and C

\subsection{A laminate optimization problem formulated on sections}

The optimization problem in this study is formulated as: given a shell-like structure partitioned to a set of geometrical regions (further on called sections) $S$, find the number and sequence and associated attributes of patches minimizing a criterion $F$. Each patch covers a connected subset of $S$ defining its shape. The connectivity of sections in $S$ can be determined from their geometrical adjacency, i.e., sections sharing geometric edges are adjacent.

As already proposed in the references cited above, graph operations enable for an elegant way to formulate the optimization problem. Therefore, we introduce a section-graph $\mathcal{G}=(S, E)$ as an abstract model of the structure to optimize. The section-graph $\mathcal{G}$ is an undirected graph with the set of sections $S$ building its vertices. Geometrically adjacent sections are connected by edges $E$ (see Fig. 3).

Moreover, we consider the $i$ th patch to be defined by its shape $\mathcal{P}_{i}$, a material $m_{i}$ from a list of available materials, an orientation in the form of a rotation angle $\phi_{i}$ with respect to a reference orientation, and a number of ply replicates

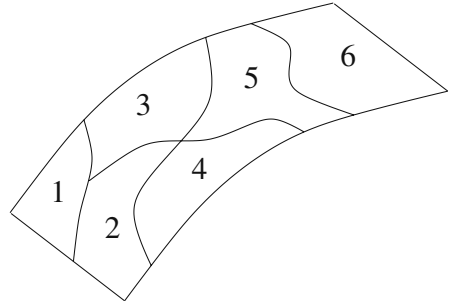

(a) Geometry

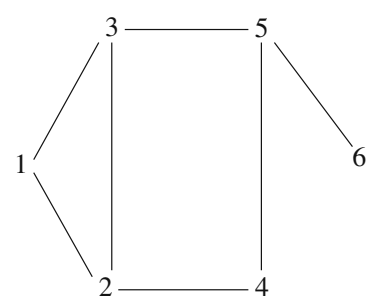

(b) Section-graph
Fig. 3 Geometry and corresponding section-graph of a curved panel partitioned to six sections

$t_{i}$. The actual thickness of the patch then corresponds to $t_{i}$ multiplied by the raw thickness of the ply material $m_{i}-$ a concession to manufacturing, where the thickness of a reinforcement treatment can only vary in discrete steps.

Hence, the laminate optimization problem can be formulated as:

$\min _{\mathbf{x} \in\left\{\left\langle\mathcal{P}_{i}, m_{i}, \phi_{i}, t_{i}\right\rangle^{n}\right\}} F(\mathbf{x})$

subject to:

$n \in \mathbb{Z}, n_{\min } \leq n \leq n_{\max } \quad$ (number of patches)

$\mathcal{P}_{i} \subset \mathcal{G}, \mathcal{P}_{i}$ connected $\quad$ (subgraph of $\mathcal{G}$ )

$m_{i} \in\left\{M_{0}, \ldots, M_{m}\right\} \quad$ (ply material)

$\phi_{i} \in \mathbb{R},-90^{\circ}<\phi_{i} \leq 90^{\circ} \quad$ (ply angle)

$t_{i} \in \mathbb{Z}, 1 \leq t \leq t_{\max }$

(number of ply replicates)

This problem formulation has some notable traits: The representation is of variable-length, i.e., the number of dimensions $n$ in the search space is not a priori defined but a variable to be optimized. The search space is structured in blocks of equal form, namely tuples $\left\langle\mathcal{P}_{i}, m_{i}, \phi_{i}, t_{i}\right\rangle$ representing one reinforcement patch each. The sequence of patches in $\mathbf{x}$ is sensitive to ordering, since its order determines the stacking sequence in the laminate. The patch attributes are heterogeneous, i.e., graph-, string-, real-, and integer-valued.

An analogous, fixed-length problem could be found by introducing $n_{\max }$ virtual patches and parameterizing their physical existence either by an additional boolean value or by allowing a zero thickness. As shown in Giger (2007) this approach would suffer from a potentially large number of variables representing attributes of physically inexistent patches. These so-called non-coding regions would participate in the optimization process without affecting the 
objective. Hence, they could affect the performance of the optimization method especially if there is a considerable difference between $n_{\min }$ and $n_{\max }$.

For the following discussion we introduce the notation $|\mathbf{x}|_{P}$ to designate the number of plies in a candidate solution and $x_{i}$ for its $i$ th ply, i.e., $\mathbf{x}=\left\{x_{0}, x_{1}, \ldots, x_{n-1}\right\}$ and $n=$ $|\mathbf{x}|_{P}$.

In order to ensure the cohesion of the structure, the design is covered by a very compliant layer. This approach is borrowed from earlier investigations (Giger et al. 2008). The properties of this compliant layer are given for the following examples. In order to minimize the influence of the fill layer on the simulated structural response, its stiffness and mass properties are set as low as possible. A lower bound is set by the condition of the finite element system, i.e., too low values may induce numerical instabilities.

\subsection{Section categories}

In order to develop operations modifying the shape of single patches, we first characterize the sections in the patch graph $\mathcal{P}_{i}$ of a single patch $i$. Figure 4 illustrates a rectangular panel partitioned into an irregular set of sections indicated by thin

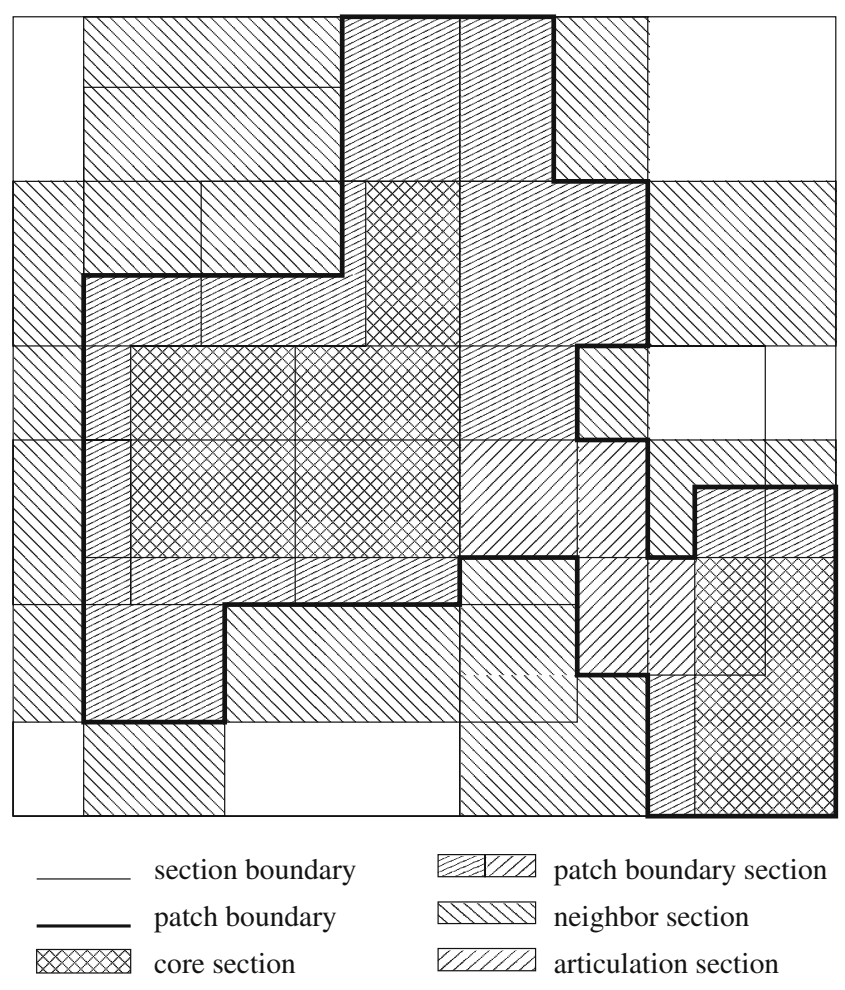

Fig. 4 Illustration of different types of sections in a structure with non-regular sections and a single patch solid lines. The panel is reinforced by a single patch inside the region surrounded by the thick solid line. Based on their adjacency, the different sections can be classified. Therefore, we introduce the notation for some common graph functions: $\mathrm{v}(G)$ returns the set of vertices in a graph $G$, $\mathrm{v}_{\text {adj }}(v, G)$ returns the set of vertices adjacent to vertex $v$ in a graph $G$, and $\mathrm{v}_{\text {art }}(G)$ returns the articulation vertices (also called cut vertices) of a graph $G$. The set of patch sections, i.e., all sections contained in the $i$ th patch is written as:

$\left\{v_{\text {patch }}\right\}=\left\{v \in \mathrm{v}\left(\mathcal{P}_{i}\right)\right\}$

The set of sections adjacent to patch $i$ can be written as

$$
\begin{aligned}
\left\{v_{\text {neighbor }}\right\}= & \left\{v \in \mathrm{v}(\mathcal{G}): v \notin \mathrm{v}\left(\mathcal{P}_{i}\right),\right. \\
& \left.\mathrm{v}_{\text {adj }}(v, G) \cap \mathrm{v}\left(\mathcal{P}_{i}\right) \neq \varnothing\right\}
\end{aligned}
$$

And the set of section on the boundary of it is computed from

$$
\begin{aligned}
\left\{v_{\text {boundary }}\right\}= & \left\{v \in \mathrm{v}\left(\mathcal{P}_{i}\right),\right. \\
& \left.\operatorname{vadj}_{\text {adj }}(v, G) \cap\left\{v_{\text {neighbor }}\right\} \neq \varnothing\right\}
\end{aligned}
$$

The set of articulation sections $\left\{v_{\text {articulation }}\right\}$ is a subset of the boundary sections: The removal of one articulation section splits $\mathcal{P}_{i}$ into two or more components.

$\left\{v_{\text {articulation }}\right\}=\operatorname{vart}_{\text {art }}\left(\mathcal{P}_{i}\right)$

\subsection{Section rankings with gradient information}

Gradient information is used to indicate whether a section's thickness has to be increased or decreased. Therefore, each section $j$ in $S$ is accompanied by a scalar parameter $\tau_{j}$ called the section's thickness multiplier. In the analysis model the ply thickness of each ply covering section $j$ is multiplied by $\tau_{j}$ which finally multiplies the laminates thickness by $\tau_{j}$. By setting the section multiplier $\tau_{j}$ to 1 does not actually change the physical expression of the laminate but nonetheless allows for the computation of the derivative $\nabla_{\tau} F(\mathbf{x})=\left[\partial F(\mathbf{x}) / \partial \tau_{j}\right]$. The number of sections is constant during the optimization and typically considerably smaller than the number of finite elements in the analysis model. The computation of these derivatives can be done by sensitivity analysis at an affordable additional cost (Mateus et al. 1991, 1997; Lund 1994; van Keulen et al. 2005).

The information available from these section thickness derivatives is injected into genetic operators locally modifying the laminate. We introduce $\operatorname{rank}(e, \mathbf{e})$ as an expression for the index of item $e$ in a sequence $\mathbf{e}$ ordered in ascending order. rank $(e, \mathbf{e})$ is an integer number in the interval 
$[0,|\mathbf{e}|-1]$. A rank value of zero indicates that $e$ has the lowest value in $\mathbf{e}$ whilst a rank $|\mathbf{e}|-1$ designates the highest value. The section rank of section $j$ is then computed as:

$\varrho_{j}=\operatorname{rank}\left(\frac{\partial F(\mathbf{x})}{\partial \tau_{j}}, \nabla_{\tau} F(\mathbf{x})\right)$

Sections with low (thus potentially negative) thickness derivatives have low ranks $\varrho_{j}$. Hence, increasing the thickness of a section with low rank will lower (or at least only little increase) the objective of a candidate solution $\mathbf{x}$. The same holds for decreasing the thickness of sections with high rank.

Thus, section rankings can indicate a tendency on where reinforcements in the form of the considered patches shall be applied or where these reinforcements shall be removed. However, they depend on the local laminate already existing in the corresponding section. Thus, section rankings may miss-estimate the influence of a reinforcement treatment if there are significant differences in the homogenized properties of the existing laminate and a potential reinforcement patch. Hence, section rankings are embedded in stochastic operations. There, they shall bias operations modifying the patch shapes without affecting the global search properties of the EA scheme.

\subsection{Basic operations on the patch shape}

Based on the above introduced section rankings basic operations modifying patch shapes can be developed. Add one section (Algorithm 2) adds a new neighbor section to an existing patch shape. The choice on which section to add is based on section rankings. The exponent in the power law (State 3) influences the balance between a random and a deterministic choice: an exponent of zero corresponds to a uniform random selection. For this study the exponent is set to 5 .

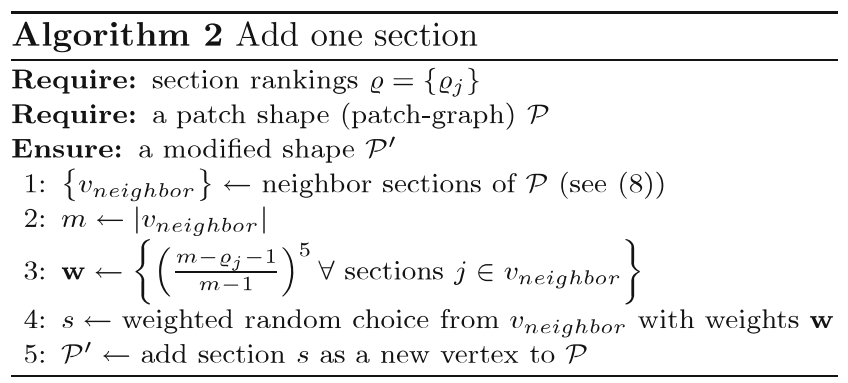

Remove one section (Algorithm 3) removes a boundary section from an existing patch shape. Articulation sections are not considered for removal in order to keep the patch graph connected. The choice on which section to remove is again based on section rankings. The power law (State 5) to compute the selection weights is complementary to the one in Algorithm 2.

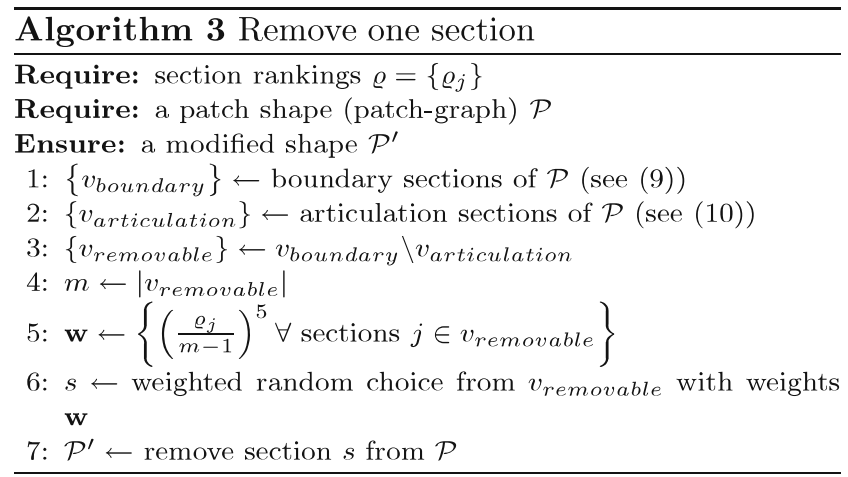

\subsection{Genetic variation operators}

Typical genetic variation operators inherit from either crossover or mutation. In most common implementations, crossover is a binary operator recombining genetic information of two parent individuals and creating two offspring. And mutation is an unary operation creating one offspring from one parent by adding some random perturbation.

The aforementioned traits of the investigated genetic representation influence the choice and design of variation operators applicable to it. Since it is structured on two different hierarchical levels-namely a laminate level and a patch level-there have to be variation operators changing information on both levels. The heterogeneous, variable-length genotype is not suitable for standard mutation and crossover operations available for bitstrings or real-valued encodings. In order to have a complete representation, all attributes of the genotype need to participate in the variation. A set of only two variation operators like in conventional EAs would hardly enable for non-disruptive changes in all attributes of parent solutions (local and Lamarckian property, see Gen and Cheng 2000). Thus, there is a set of different genetic operations for each level and attribute-type. Variation operators are applied one at the time on the selected parents according to the application rates $r_{i}$ as indicated in Fig. 1 and Algorithm 1.

The following operators modify information on the laminate level: Stacking mutation changes the stacking sequence in a laminate Algorithm 4. Add patch mutation adds a new patch to the laminate (Algorithm 5). Remove patch mutation removes a patch from the laminate (Algorithm 6). Split-andsplice patch crossover cuts the laminate stack of two parent solutions each at a random position and recombines the head and tail segments to two new offspring. 

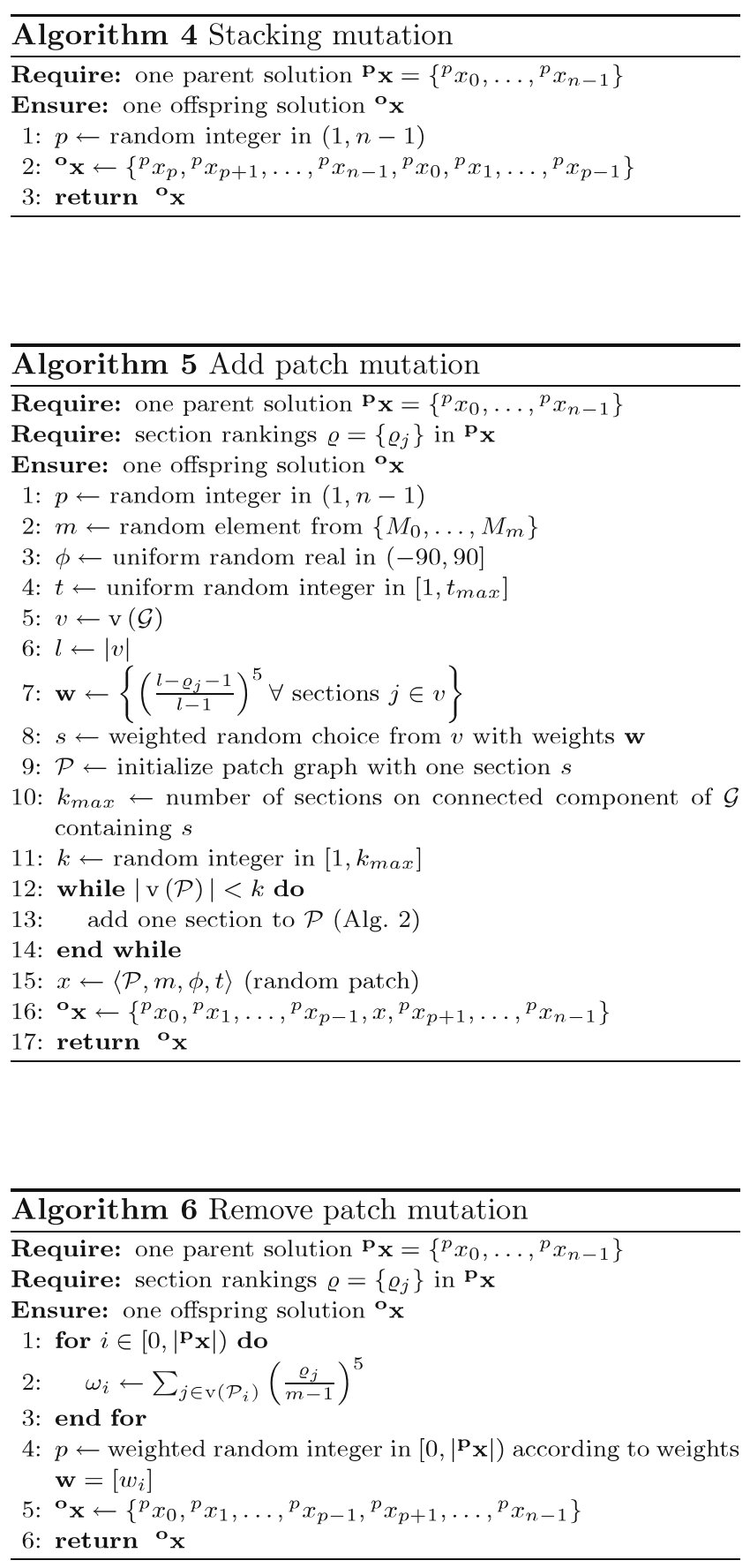

The following operators act on the patch level: Material mutation selects a random patch from a parent solution and creates one offspring by randomly exchanging the material attribute of the selected patch. Gaussian ply-angle mutation changes the ply-angles of all patches in a parent solution by adding a random value from $\mathcal{N}\left(\mu=\phi_{i}, \sigma=5^{\circ}\right)$. SRIply-angle mutation uses a symmetric rank-1-updating (a deterministic quasi Newton method) to locally optimize the ply-angles in a parent solution. This operator requires by itself a sequence of evaluations. This is enabled by the parallel execution of the variation state in the employed

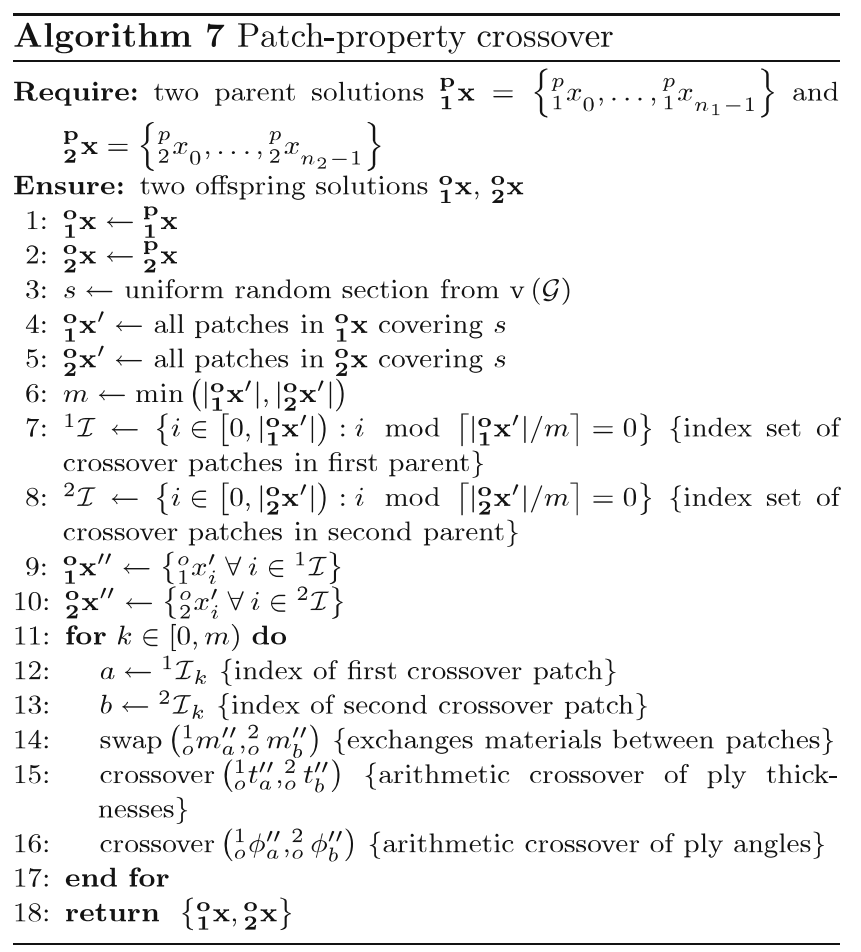

asynchronous EA scheme. Patch-thickness mutation sets the patch thickness of a random patch in a parent solution to a random integer in $\left[1, t_{\max }\right]$. Grow-patch mutation selects a random patch in a parent solution and adds an additional section to the patch shape by Algorithm 2. Shrink-patch mutation selects a random patch in a parent solution and removes a section from the patch shape by Algorithm 3 . Move-patch mutation selects a random patch in a parent solution. Then the patch shape is modified by first applying Algorithm 2 and later Algorithm 3. Thus, it adds one section and removes potentially another one, i.e., it changes the location of reinforcement patch. Patch-property crossover recombines patch attributes in two parent solutions (Algorithm 7). This operator exchanges ply materials and employs arithmetic crossovers on ply thicknesses and ply-angles (see e.g., König 2004). In general, the two parents $\left({ }_{1}^{\mathbf{p}} \mathbf{x},{ }_{2}^{\mathbf{p}} \mathbf{x}\right)$ have not the same length. Then, the patches exchanging genetic information do not map one-to-one. Hence, all patches of the shorter parent but only a subset of the longer parent are candidates to exchange genetic information. Furthermore, the sensitivity of the encoding to the ordering information has to be respected. The mechanism is given in detail in Algorithm 7. An illustration for an example case is depicted in Fig. 5.

Fig. 5 Crossover pairings for parents of different length in Algorithm 7

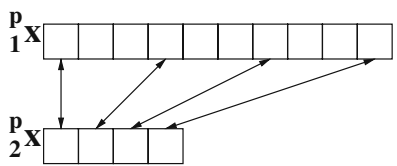




\section{Implementation}

The method is implemented in an object-oriented code in the Python programming language ${ }^{1}$ (version 2.5). Grid Engine $6 \mathrm{NI}^{2}$ is employed as a queuing system on a heterogeneous cluster consisting of 28 blade computers and two workstations with two CPUs each. MySQL 5.0 $0^{3}$ serves as a database server, InnoDB ${ }^{4}$ is used as underlaying storage engine. The database service as well as the scheduler run on the same additional server computer. The commercial finite element code ABAQUS (version 6.8) is employed for the structural simulations. Sensitivities are computed by a direct differentiation scheme (see Section 2.18 in ABAQUS 2008). The implementation operates on ABAQUS-input files and recognizes user defined element sets as sections. The sectiongraph is constructed automatically from meshed shell structures with arbitrary geometry. A set of naming conventions and regular expressions is employed to map candidate solutions back to valid ABAQUS-input files. Special mapping procedures for geometrically symmetric structures, regions excluded from optimization, or symmetric laminates are available. The lamination plan of each candidate solution is fully parameterized in the corresponding input file.

\section{Numerical experiments}

\subsection{Single-patch vibrating plate}

A rectangular plate (Fig. 6) with an additional, non-central mass shall be optimized for its first natural frequency $f_{0}$, subject to an upper limit constraint on the overall structural mass $\mathcal{M} \leq \mathcal{M}_{\text {max }}$. The structure is covered by a very compliant fill-material (isotropic, Young's modulus $E=1 \mathrm{GPa}$, Poisson's ratio $v=0.3$, thickness $0.15 \mathrm{~mm}$, area density $0.237 \mathrm{~kg} / \mathrm{m}^{2}$ ). The shaded region in Fig. 6 is covered by an additional mass layer (isotropic, $E=1 \mathrm{GPa}, v=0.3$, thickness $10 \mathrm{~mm}$, area density $158 \mathrm{~kg} / \mathrm{m}^{2}$ ). In a first experiment, the structure shall be reinforced by a single, additional layer of an unidirectional carbon/epoxy prepreg (Table 1, thickness $0.15 \mathrm{~mm}$ ). The experiment corresponds to problem 5.3.1 presented in Giger (2007) (revisited in Section 7.5 in the same source and also presented in Giger et al. 2008).

The original optimization problem is formulated as:

$$
\max _{\mathbf{x}} f_{0}(\mathbf{x})
$$

subject to $\mathcal{M}(\mathbf{x})-\mathcal{M}_{\max } \leq 0$

\footnotetext{
${ }_{1}^{1}$ http://www.python.org

${ }^{2} \mathrm{http}: / /$ gridengine.sunsource.net

${ }^{3} \mathrm{http}: / /$ www.mysql.com

${ }^{4}$ http://www.innodb.com
}

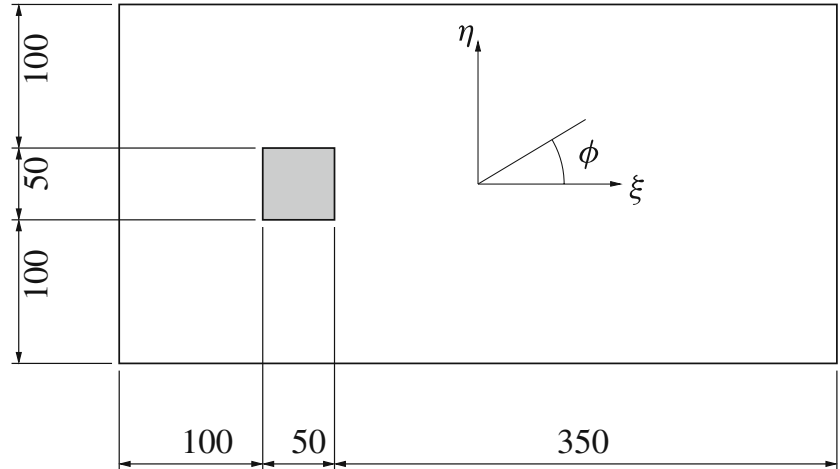

Fig. 6 Geometry of the vibrating plate experiment: the rectangular plate is simply supported on all edges. The shaded region corresponds to an additional mass. All dimensions are in $\mathrm{mm}$

An exterior penalty method and scaling is employed in order to get an unconstrained, scalar valued fitness function (e.g., Bertsekas 1982):

$\min _{\mathbf{x}} F(\mathbf{x})=-\frac{f_{0}(\mathbf{x})}{f_{s}}+p\left[\max \left\{0, \frac{\mathcal{M}(\mathbf{x})-\mathcal{M}_{\max }}{\mathcal{M}_{s}}\right\}\right]^{2}$

On the constraint (i.e., when $\mathcal{M}(\mathbf{x})=\mathcal{M}_{\text {max }}$ ) the limit value from the forbidden side of the constraint is taken as derivative of $F$ with respect to decision variables in $\mathbf{x}$. For the investigated problem, the values are set to $\mathcal{M}_{\max }=$ $0.436475 \mathrm{~kg}, \mathcal{M}_{s}=0.05 \mathrm{~kg}, p=10$, and $f_{s}=2 \mathrm{~Hz}$. A reachable design identified by prior experiments has a natural frequency in the range of $2 \mathrm{~Hz}$. This motivates the scaling factor $f_{s}$. Some experiments lead to the choice for the scaling and penalty factor for the mass constraint. The number of patches $(n)$ is restricted to one $\left(n_{\min }=n_{\max }=\right.$ 1 ), the ply material list contains only the above characterized unidirectional prepreg, and the number of raw material layers in the reinforcement is set to one $\left(t_{\min }=t_{\max }=1\right)$. An unbiased setup of fifty sections in a regular grid of five along the short and ten along the long side of the plate (cf. Fig. 8) is chosen. The finite element model contains 200 four-node, layered-shell elements in a square shape, thus, each section is modeled by four elements.

Ten independent optimization runs are carried out. For each run, the pool size is set to 250 individuals, four demes are employed, and migration frequency is set to 750 evaluations. The application rates for the operators are set to $90 \%$ for Patch-property crossover, $2 \%$ for Grow-patch-mutation, $2 \%$ for Shrink-patch-mutation, $2 \%$ for Move-patch mutation, $2 \%$ for Gaussian ply-angle mutation, 2\% SR1-plyangle mutation, and zero for the remaining operators. A pool of 250 individuals is initialized by random for each run. The optimization is stopped after 15,000 evaluations. 
Results and discussion Convergence plots for the first eigenfrequency and the mass are given in Fig. 7. There, it can be seen that from around 7,000 evaluations on, no run is able to improve its best solution. As can be expected, all allowed mass is invested in a reinforcement layer and hence all solutions found are on the constraint. Moreover, nine out of ten runs find a solution with an eigenfrequency in a range between 2.036 and $2.047 \mathrm{~Hz}$, only one run gets stuck at $1.928 \mathrm{~Hz}$.

The shape of the single reinforcement patch for the best individual of each run is shown in Fig. 8. Although the geometry of the structure has a horizontal symmetry, only one reinforcement layer shows this symmetry (Fig. 8h) with

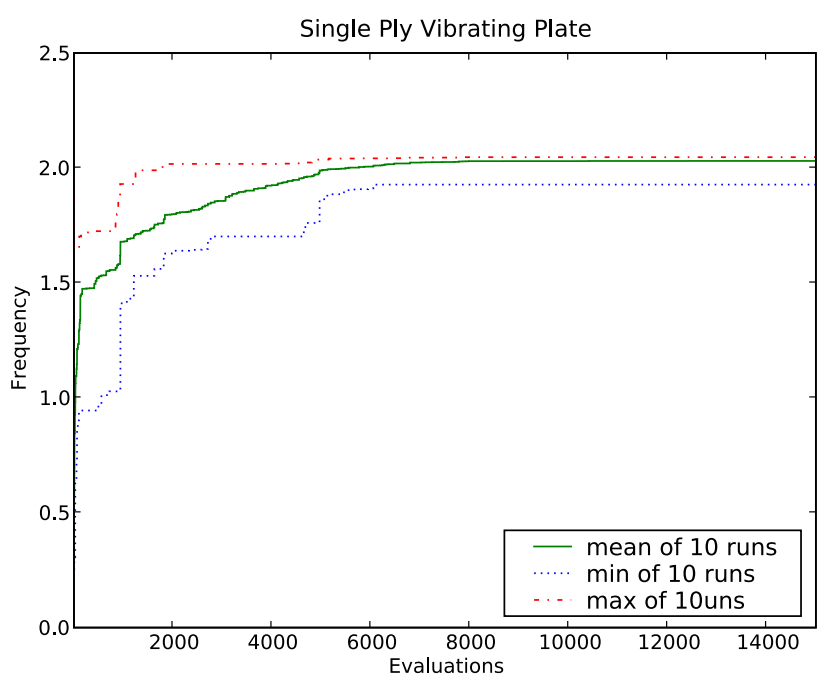

(a) Frequency of best individual averaged over 10 runs

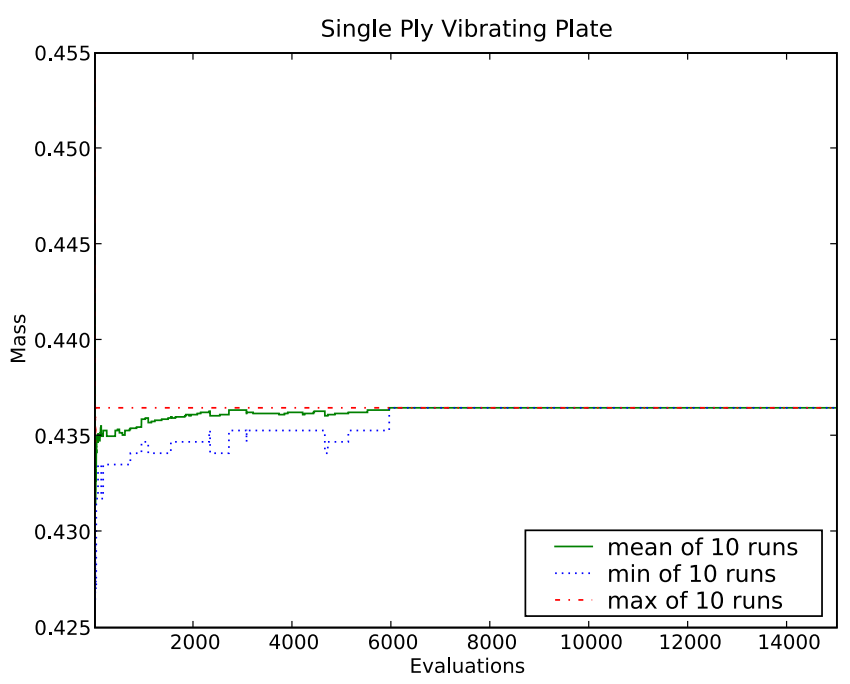

(b) Mass of best individual averaged over 10 runs

Fig. 7 Results of the single-ply vibrating plate experiment

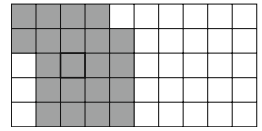

(a) $f_{0}=2.042 \mathrm{~Hz}$ $\mathcal{M}=0.436 \mathrm{~kg}$,

$\phi=-88.51^{\circ}$

(d) $f_{0}=2.042 \mathrm{~Hz}$, $\mathcal{M}=0.436 \mathrm{~kg}$, $\phi=87.91^{\circ}$

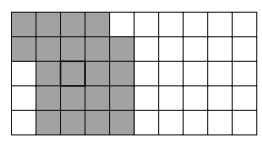

(g) $f_{0}=2.042 \mathrm{~Hz}$, $\mathcal{M}=0.436 \mathrm{~kg}$, $\phi=-87.90^{\circ}$

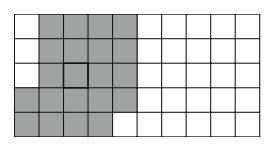

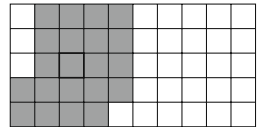

(b) $f_{0}=2.042 \mathrm{~Hz}$, $\mathcal{M}=0.436 \mathrm{~kg}$, $\phi=88.36^{\circ}$

(e) $f_{0}=2.046 \mathrm{~Hz}$, $\mathcal{M}=0.436 \mathrm{~kg}$, $\phi=88.23^{\circ}$

(h) $f_{0}=1.928 \mathrm{~Hz}$, $\mathcal{M}=0.436 \mathrm{~kg}$, $\phi=89.95^{\circ}$

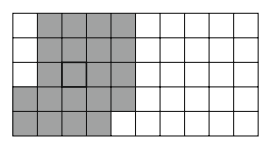

(j) $f_{0}=2.042 \mathrm{~Hz}$, $\mathcal{M}=0.436 \mathrm{~kg}$, $\phi=88.15^{\circ}$
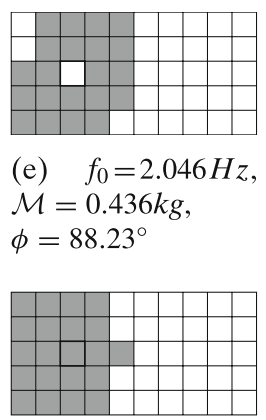

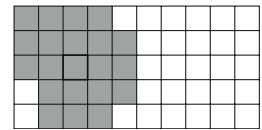

(c) $f_{0}=2.036 \mathrm{~Hz}$, $\mathcal{M}=0.436 \mathrm{~kg}$, $\phi=89.84^{\circ}$

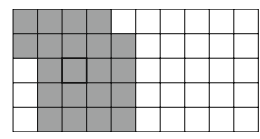

(f) $f_{0}=2.040 \mathrm{~Hz}$, $\mathcal{M}=0.436 \mathrm{~kg}$, $\phi=-89.92^{\circ}$

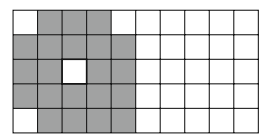

(i) $f_{0}=2.047 \mathrm{~Hz}$, $\mathcal{M}=0.436 \mathrm{~kg}$, $\phi=-88.62^{\circ}$
Fig. 8 Best individuals identified in ten different runs for the singleply vibrating plate experiment with their first eigenfrequency $f_{0}$, mass $\mathcal{M}$, and ply-angle $\phi$. Reinforced sections are shaded

a symmetric patch shape and a ply-angle of approximately $90^{\circ}$. Interestingly, this solution has the lowest eigenfrequency within the ten best solutions. Given the horizontal symmetry, six out of ten solutions have the same patch shape (Fig. 8a, b, d, f, g, and j). Their ply-angles are in range of $87.91-89.92^{\circ}$ and the corresponding eigenfrequency ranges from 2.040 to $2.042 \mathrm{~Hz}$. The two best solutions (Fig. 8e and i) feature a hole in the patch shape where the mass layer is situated. This allows for making use of the stiffness of the mass layer and at the same keeping the mass in this critical region at the lowest value possible. Since there is no operator inserting holes in a patch shape, the patch has just grown around the mass section for these two runs.

\subsection{Twelve-patch vibrating plate}

The vibrating plate experiment is repeated with the following changes: a variable number of patches is allowed in an interval $1 \leq n \leq 12$. The maximum number of ply replicates is set to $t_{\max }=3$. The fitness function (14) is adapted to the heavier structure by setting $f_{s}=30 \mathrm{~Hz}$, $\mathcal{M}_{\max }=0.543125 \mathrm{~kg}$, and $\mathcal{M}_{s}=0.05 \mathrm{~kg}$. The number of 


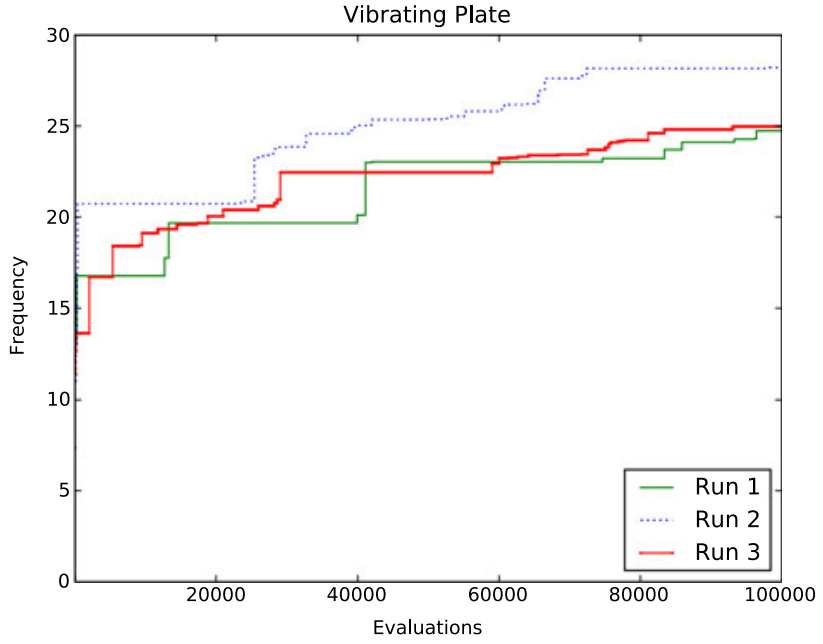

(a) Frequency $f_{0}$

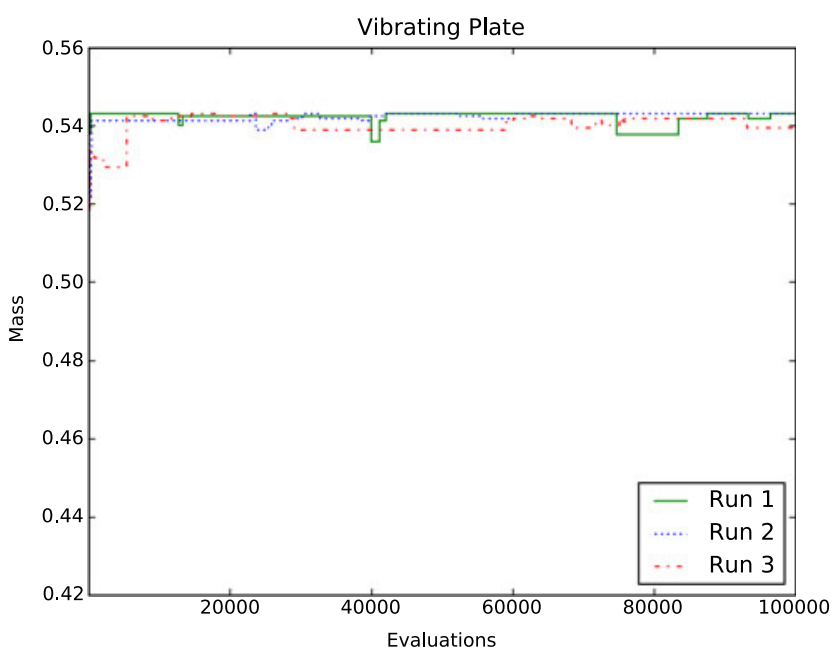

(b) Mass $\mathcal{M}$

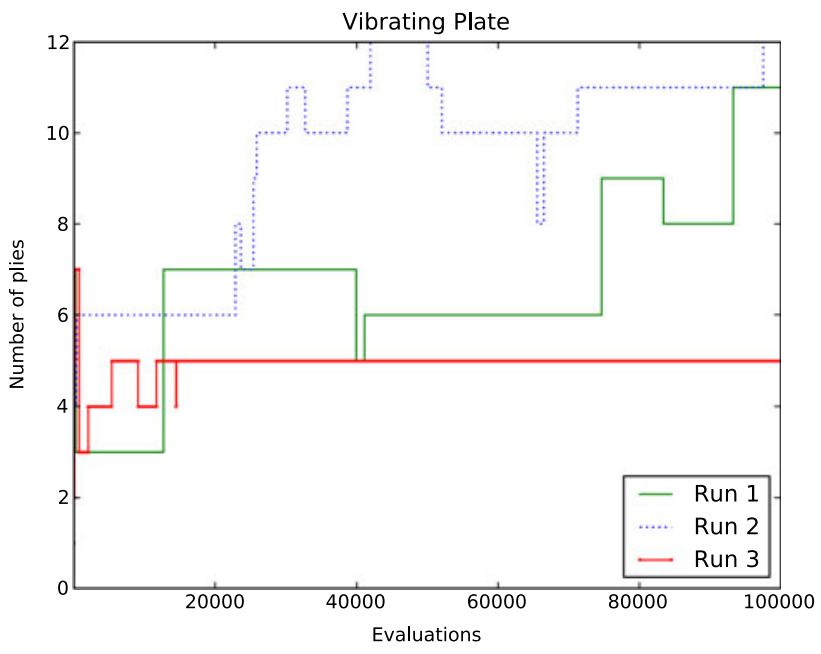

(c) Number of plies $|\mathbf{x}|_{P}$

Fig. 9 Convergence plots for the twelve-ply vibrating plate experiment for all three runs patches and the corresponding value of the mass constraint are taken from Giger (2007). The scaling factor $f_{s}$ is motivated by reachable designs with its first natural frequency in the range of $25-30 \mathrm{~Hz}$ identified in previous experiments. The scaling factor $M_{s}$ stays the same as in the single patch experiment (Section 4.1).

The variation operator rates are set to: $50 \%$ Split-andsplice patch crossover, $40 \%$ Patch-property crossover, and $1 \%$ for each of the remaining operators. In order to cope with the larger search space the pool size is increased to 400 individuals in 16 demes, the migration frequency is set to 1,200 . The initial pool is generated randomly. Three independent runs are carried out for 100,000 evaluations each.

Results and discussion Convergence plots are given in Fig. 9. The plies of the best solution of all runs (run 2) are illustrated in Fig. 10. The best solution reaches an eigenfrequency of $28.214 \mathrm{~Hz}$ at a mass of $0.543125 \mathrm{~kg}$. The remaining runs find a solution with $f_{0}=24.7396, \mathcal{M}=$ $0.543125 \mathrm{~kg}$ and $f_{0}=24.9772 \mathrm{~Hz}, \mathcal{M}=0.53957 \mathrm{~kg}$, respectively.

The convergence plots in Fig. 9 show little improvements over the last evaluations but not yet a stagnation after 100,000 function evaluations. Hence, the runs may not be

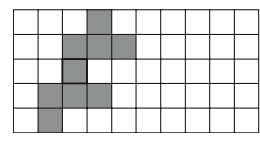

(a) Ply $1: \phi=$ $64.99^{\circ}, t=2$

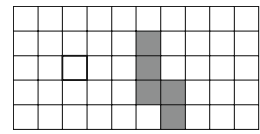

(d) Ply $4: \phi=$ $-72.93^{\circ}, t=2$

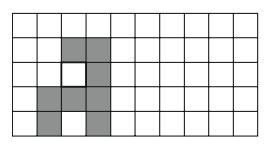

(g) Ply $7: \phi=$ $-80.74^{\circ}, t=1$

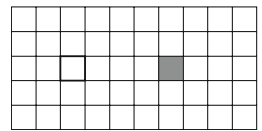

(j) Ply $10: \phi=$ $-80.85^{\circ}, t=2$

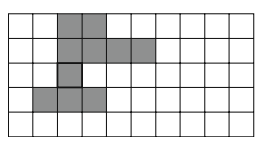

(b) Ply $2: \phi=$ $-87.31^{\circ}, t=1$

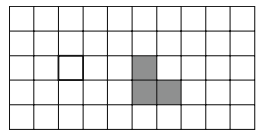

(e) Ply $5: \phi=$ $-75.32^{\circ}, t=2$

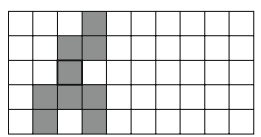

(h) Ply $8: \phi=$ $-80.73^{\circ}, t=1$

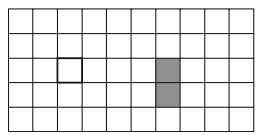

(k) Ply $11: \phi=$ $-80.85^{\circ}, t=2$

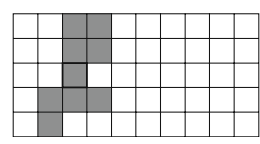

(c) Ply $3: \phi=$ $-71.47^{\circ}, t=1$

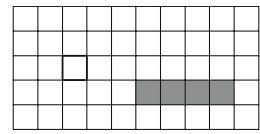

(f) Ply $6: \phi=$ $-80.39^{\circ}, t=2$

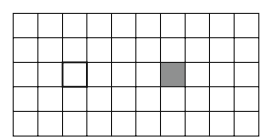

(i) Ply 9 : $\phi=-80.85^{\circ}$, $t=1$

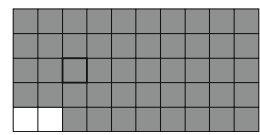

(1) Ply $12: \phi=$ $-86.10^{\circ}, t=1$
Fig. 10 Plies of the best individual for the twelve-ply vibrating plate experiment with their corresponding ply angles $\phi$ and thickness multipliers $t$. Reinforced sections are shaded 
fully converged. As expected, the mass constraint is reached by all solutions. The patch shapes as well as the angles shown in Fig. 10 do not allow for an intuitive interpretation. However, as already observed in Giger (2007) the reinforcement do not only concentrate on the mass region but also the right half of the structure. This is due to the fact that reinforcing the mass region will shift the first eigenmode to the yet weaker right half of the structure. The asymmetry with respect to the geometric symmetry axis may be explained by a kind of a self-excited instability: once an asymmetric reinforcement is applied, there is potentially no longer a pressure to remove this asymmetry. Further reinforcements will even be shifted to exploit it. Hence, the effect already observed in the single-ply experiment (Section 4.1), where a majority of the runs found an asymmetric patch geometry seems to be amplified in the investigated thicker laminates.

\section{Application example: mass optimization of an airplane side rudder}

An airplane's side rudder is a control surface attached to the vertical tail and used to initiate a yaw moment. The

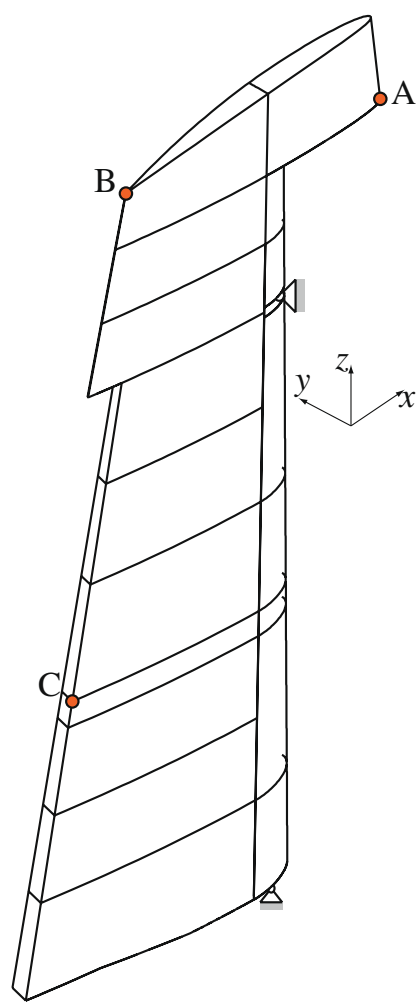

(a) Isometric view

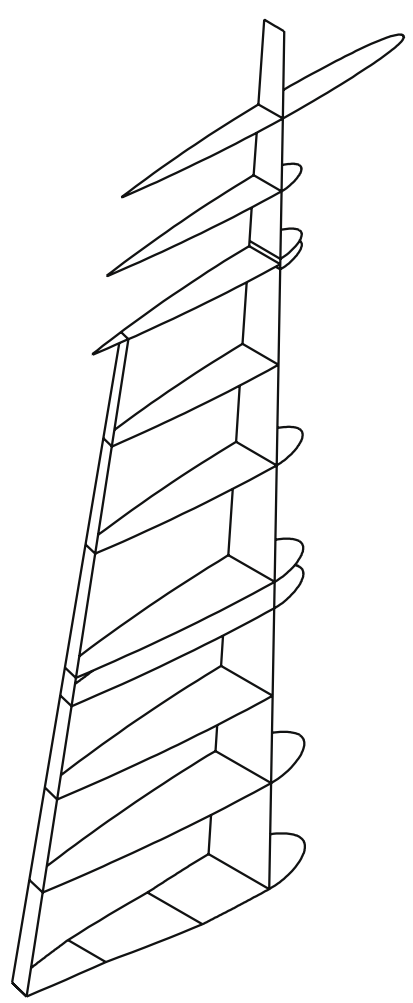

(b) Spar and ribs
Fig. 11 Aircraft side rudder. The rudder is attached to the vertical tail whereas the upper attachment point enables for rotations around the vertical axis, the lower support is used to introduce the steering moment (and is hence fixed in the analysis) attachment allows for rotations around the aircraft's yaw axis (Fig. 11). For the investigated component of a short range aircraft the steering moments are induced into the nose part of the bottom rib. The second attachment point is realized by two short supports on the eighth rib.

The rudder is subject to aerodynamic forces acting on its skin. An additional, non-structural mass is positioned in the front top section of the structure to balance steering reactions. Depending on the flight state, this mass induces considerable forces of inertia.

The structure is built from a single spar and ten ribs supporting the aerodynamic active skin. The trailing edge gives support to a trim-rudder. The structure is nearly symmetric with respect to the $x$-z-plane. A slight asymmetry is induced by the trailing edge which is inclined to the port side to give place for the trim-rudder attachment and actuation devices.

For the analysis the different distributed and concentrated loads of a dimensioning flight state are reduced to a set of line-distributed forces and moments (Shear-Moment-andTorque, SMT) on the wing-box centerline. From there, they are summed up to the rib positions and introduced into each rib's center point. These non-structural points are connected by rigid body elements to the rib's boundary (for a detailed description of the SMT-method see e.g., Appendix A in Ledermann 2006). The resulting displacement field for the considered load case is a superposition of a torsional deformation around the $z$-axis and two-point bending between the support points.

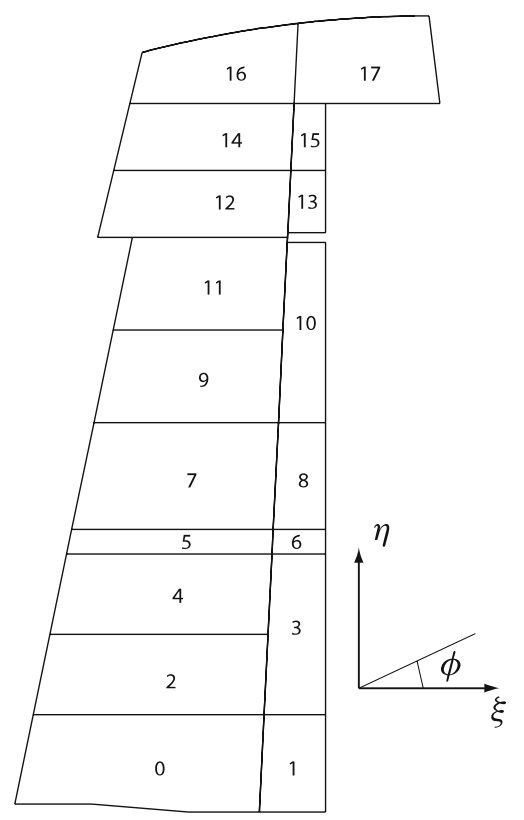

Fig. 12 The skin of the rudder is partitioned to 36 lamination sections. The laminate of the 18 sections illustrated here is mirrored at the $x$ $z$-plane to the second half-shell. The reference coordinate system $\xi-\eta$ with angle $\phi$ is used to determine the fiber direction 
A composite structure shall be compared to a reference metal construction manufactured from aluminum. The fiber reinforced component shall be optimized for mass subject to constraints on strength and stiffness, i.e., the displacements at three reference points (A, B, and C in Fig. 11a) shall be at most equal to the ones of the reference construction. The reference points are positioned such that both bending and torsional displacements can be captured. The optimization problem is formulated as:

$$
\min _{\mathbf{x}} \mathcal{M}(\mathbf{x})
$$

subject to: $\left\|\tilde{u}_{A}(\mathbf{x})\right\|-\left\|\tilde{u}_{A, \text { ref }}\right\| \leq 0$

$\left\|\tilde{u}_{B}(\mathbf{x})\right\|-\left\|\tilde{u}_{B, \text { ref }}\right\| \leq 0$

$\left\|\tilde{u}_{C}(\mathbf{x})\right\|-\left\|\tilde{u}_{C, \text { ref }}\right\| \leq 0$

$$
\max _{\Omega}\left\{i_{\text {TsaiHill }}(\mathbf{x})\right\}-1 \leq 0
$$

Where $\mathcal{M}$ denotes the structural mass and $\|\tilde{u}\|$ a displacement magnitude in the specified reference point. The TsaiHill failure criterion is applied to predict a first ply failure:
The criterion computes a failure index $i_{\text {TsaiHill }}$ from the direction-wise stresses in each ply. It is evaluated at every layer top and bottom interface for each integration point for each finite element in the structure $\Omega$. Failure is predicted for $i_{\text {TsaiHill }}>1$.

Scaling and an exterior penalty method are used to transform this problem to an unconstrained, scalar minimization problem:

$$
\begin{aligned}
\min _{\mathbf{x}} F(\mathbf{x})= & \frac{\mathcal{M}(\mathbf{x})}{\mathcal{M}_{\text {ref }}} \\
& +p\left[\max \left\{0, \frac{\left\|\tilde{u}_{A}(\mathbf{x})\right\|-\left\|\tilde{u}_{A, \text { ref }}\right\|}{u_{s}}\right\}^{2}\right. \\
& +\max \left\{0, \frac{\left\|\tilde{u}_{B}(\mathbf{x})\right\|-\left\|\tilde{u}_{B, \text { ref }}\right\|}{u_{S}}\right\}^{2} \\
& +\max \left\{0, \frac{\left\|\tilde{u}_{C}(\mathbf{x})\right\|-\left\|\tilde{u}_{C, \text { ref }}\right\|}{u_{S}}\right\}^{2} \\
& \left.+\max \left\{0, \frac{\max _{\Omega}\left\{i_{\text {TsaiHill }}(\mathbf{x})\right\}-1}{i_{S}}\right\}^{2}\right]
\end{aligned}
$$

The values of the metal construction are taken as a reference: These are $\mathcal{M}_{\text {ref }}=4.08 \mathrm{~kg},\left\|\tilde{u}_{A, \text { ref }}\right\|=0.6689 \mathrm{~mm}$, $\left\|\tilde{u}_{B, \text { ref }}\right\|=1.2938 \mathrm{~mm}$, and $\left\|\tilde{u}_{C, \text { ref }}\right\|=1.3665 \mathrm{~mm}$. The

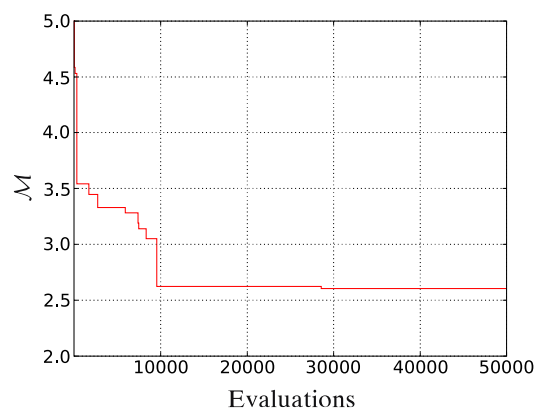

(a) Mass $\mathcal{M}$

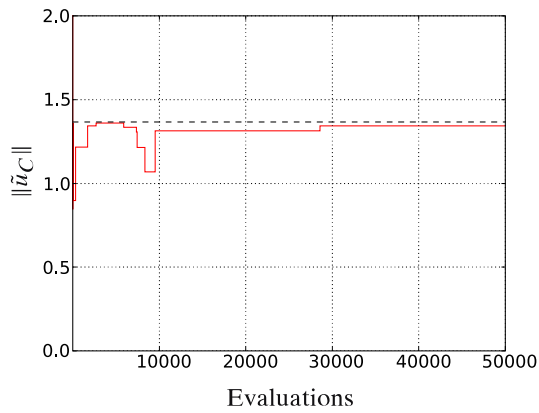

(d) Displacement $\left\|\tilde{u}_{C}\right\|$

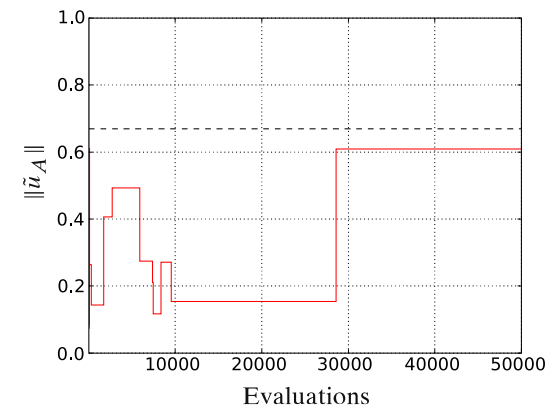

(b) Displacement $\left\|\tilde{u}_{A}\right\|$

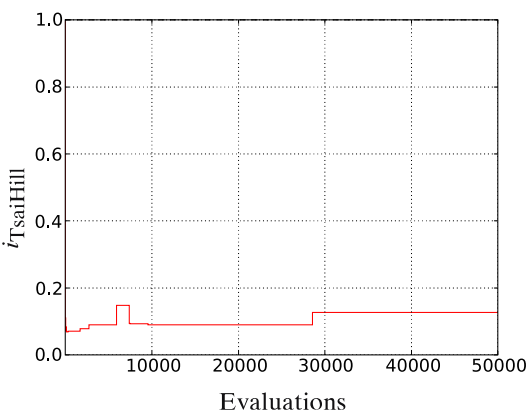

(e) Tsai-Hill failure index $i_{\text {TsaiHill }}$

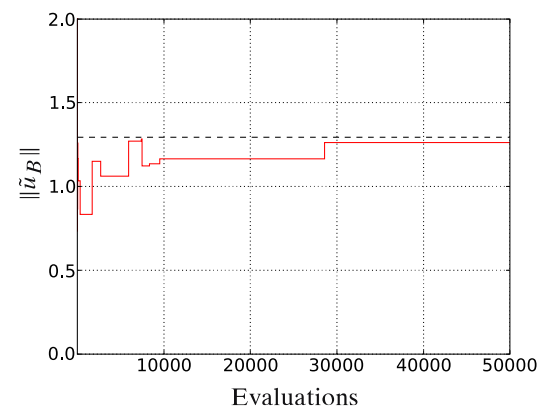

(c) Displacement $\left\|\tilde{u}_{B}\right\|$

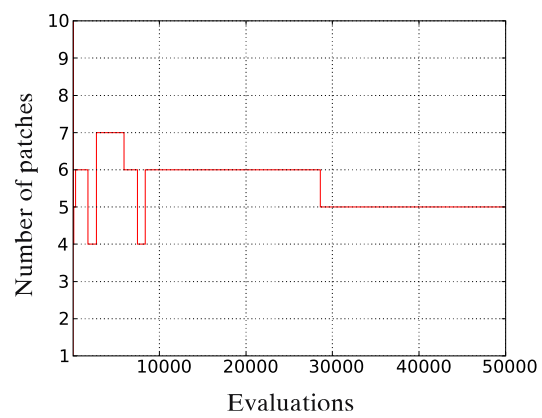

(f) Number of patches $|\mathbf{x}|_{p}$

Fig. 13 Convergence plots for the side rudder application. Mass $\mathcal{M}$, displacements $\left\|\tilde{u}_{A}\right\|,\left\|\tilde{u}_{B}\right\|$, and $\left\|\tilde{u}_{C}\right\|$, Tsai-Hill failure index $i_{\text {TsaiHill }}$ and number of patches of the best solution in the pool as a function of the number of evaluations 
scaling values are set to $u_{s}=10^{-3} \mathrm{~mm}$ and $i_{s}=10^{-3}$. The penalty factor is set to $p=10$.

The internal structure on ribs, spar, and trailing edge are covered by laminates from carbon/epoxy prepreg material (Section 1): for the spar and the trailing edge $[ \pm 30]_{s}(0.4$ $\mathrm{mm}$ thickness, zero-direction along the vertical axis) and $[ \pm 45]_{s}$ for the ribs $(0.4 \mathrm{~mm}$ thickness). The laminate on the skin shall be optimized. It is initially covered by a ply of a very compliant, isotropic fill material (Young's modulus $E=1 \mathrm{MPa}$, Poisson's ration $v=0$, specific mass $\rho=0$ $\mathrm{kg} / \mathrm{m}^{3}$, thickness $0.01 \mathrm{~mm}$ ). The skin is partitioned to 36 sections (Fig. 12). The section properties of the 18 starboard sections are mirrored at the $x-y$-plane onto the port side in order to obtain a structure with symmetric properties.

The skin reinforcement shall consist of either material 1 (Table 1, ply thickness $0.125 \mathrm{~mm}$ ) or material 2 (Table 2, ply thickness $0.1 \mathrm{~mm}$ ). The reinforcement plies are applied symmetrically on top and bottom surfaces of the laminate in order to obtain symmetric laminates. A maximum number of ten patches shall be applied. Each patch may have a thickness of at most ten times the raw material thickness.

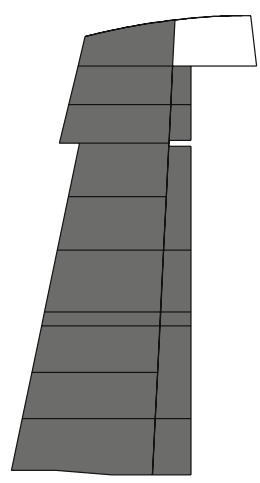

(a) Ply $1: \phi=$ $18.46^{\circ}, t=1, m=2$

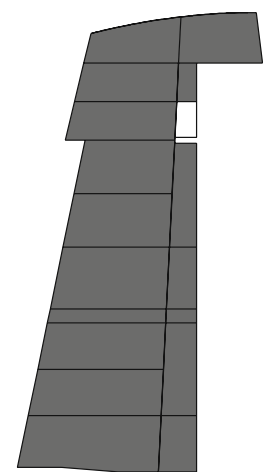

(b) Ply $2: \phi=$ $-40.79^{\circ}, t=1, m=2$

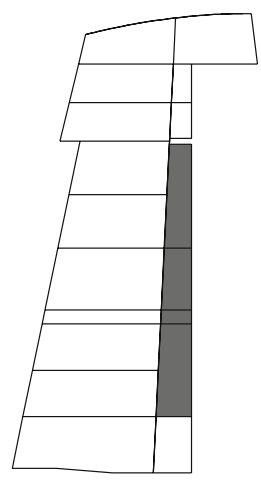

(c) Ply $3: \phi=$ $84.35^{\circ}, t=2, m=2$

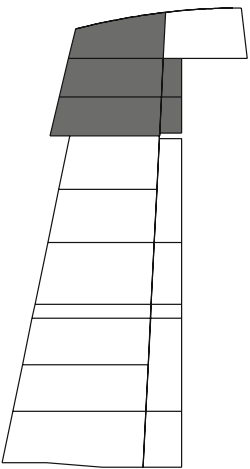

(d) Ply $4: \phi=$ $-59.47^{\circ}, t=2, m=1$

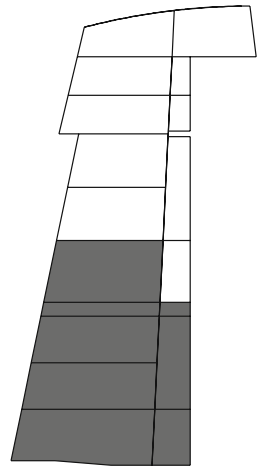

(e) Ply $5: \phi=$ $62.31^{\circ}, t=2, m=1$
Fig. 14 Plies of the side rudder optimization application with their material $m$, orientation $\phi$, and thickness multiplier $t$
Results and discussion Convergence plots are given in Fig. 13. The best solution found has a mass $\mathcal{M}$ of $2.6040 \mathrm{~kg}$. The displacement values are $\left\|\tilde{u}_{A}\right\|=0.6523 \mathrm{~mm},\left\|\tilde{u}_{B}\right\|=$ $1.2573 \mathrm{~mm}$, and $\left\|\tilde{u}_{C}\right\|=1.32595 \mathrm{~mm}$. The Tsai-Hill failure index $i_{\text {TsaiHill }}$ reaches 0.125088 . Hence, the design is in the feasible region. It is covered by five patches which are illustrated in Fig. 14.

As seen from Fig. 13, the problem is dominated by the displacement constraints. Strength does never reach a critical level for the best solution in the pool. Two plies cover almost the whole structure (Fig. 14a and b). One ply (Fig. 14c) reinforces the nose between the two support points against bending. The remaining two plies (Fig. 14d and e) reinforce the support regions. Section 17 is covered only by Ply 2 . In a practical application this issue would be eliminated if multiple load cases or additional constraints on a minimal laminate thickness (e.g., driven by damage tolerance requirements) had to be considered.

The evaluation of a single individual from given genotype information to a scalar fitness takes about three minutes. The number of parallel evaluations is mainly limited by the number of available licenses of the evaluation software in our framework. The investigated side-rudder optimization had a runtime of roughly one week.

\section{Concluding remarks}

A method for the global optimization of locally varying laminates has been investigated. It builds on a genetic representation tailored to the requirements of laminate optimization problems. The method employs graph concepts in the encoding of the geometric region covered by a reinforcement treatment. However, abandoning a purely graph-based genotype like in existing methods (Giger et al. 2008; Giger 2007; Kaufmann 2006) leads to more compact representations. The genetic search is assisted by gradient information in operations modifying the patch shape and determining optimal ply angles. The optimization method is applicable to a wide set of laminate optimization problems. Applications on eigenfrequency and stiffness- and strength-constrained mass optimization problems are presented.

The methods behavior is investigated in two academic example problems. Whilst for the first experiment (Section 4.1) the results are of reproducible quality, the more complex experiment (Section 4.2) can not confirm this assumption. However, also there, the method is able to find significantly improved designs in all runs. The spread in the single-patch experiment is considerably reduced over previous approaches presented in Giger et al. (2008).

The application to a side rudder structure illustrates a possible use-case of the method. There, an obvious partitioning of the structure into geometric sections is given by the 
construction. The method is able to find feasible solutions in this constrained problem. In the single run investigated the method finds rapid and large improvements over the first 10,000 evaluations and shows quasi-stagnation afterwards. The vertical-tail optimization presented in Giger et al. (2008) is of comparable size and complexity. However, there, the optimization discovered very small improvements over subsequent generations and showed no stagnation even after 25,000 evaluations-which is the maximum number of evaluations investigated there. The number of evaluations per unit time is roughly doubled for the side-rudder application when compared to the vertical tail optimization due to asynchronous (vs. synchronous) parallelization of the algorithm scheme.

Acknowledgments This work contributes to the project Unified Topology and Shape Design Optimization of Composite Structures gratefully supported by the Swiss National Science Foundation (SNF 200021-II 9689I1). Thanks go to Prof. Paolo Ermanni, head of the Centre of Structure Technologies, for enabling this research, to Florian Hürlimann for his help in the modeling of the airplane side rudder, and to Dr. Gerald Kress and Ursula Keller for the proofreading of the manuscript.

\section{Appendix: Material data}

The following tables list homogenized, orthotropic mechanical properties of the materials used in the numerical models (Young's moduli $E$, shear moduli $G$, Poisson's ratio $v$, specific mass $\rho$ ), strength values (tension $X_{t}, Y_{t}, Z_{t}$, compression $X_{c}, Y_{c}, Z_{c}$, shear $S$..).

Table 1 Material 1: unidirectionally reinforced carbon-epoxy prepreg

\begin{tabular}{llllll}
\hline$E_{11}$ & $135 \mathrm{GPa}$ & $E_{22}$ & $10 \mathrm{GPa}$ & $E_{33}$ & $10 \mathrm{GPa}$ \\
$G_{23}$ & $3.8 \mathrm{GPa}$ & $G_{31}$ & $5 \mathrm{GPa}$ & $G_{12}$ & $5 \mathrm{GPa}$ \\
$\nu_{23}$ & 0.3 & $\nu_{31}$ & 0.27 & $\nu_{12}$ & 0.27 \\
$\rho$ & $1,580 \mathrm{~kg} / \mathrm{m}^{3}$ & & & & \\
$X_{t}$ & $1,450 \mathrm{MPa}$ & $Y_{t}$ & $55 \mathrm{MPa}$ & $Z_{t}$ & $55 \mathrm{MPa}$ \\
$X_{c}$ & $1,400 \mathrm{MPa}$ & $Y_{c}$ & $170 \mathrm{MPa}$ & $Z_{c}$ & $170 \mathrm{MPa}$ \\
$S_{y z}$ & $90 \mathrm{MPa}$ & $S_{z x}$ & $90 \mathrm{MPa}$ & $S_{x y}$ & $90 \mathrm{MPa}$ \\
\hline
\end{tabular}

Table 2 Material 2: unidirectionally reinforced carbon-epoxy prepreg

\begin{tabular}{llllll}
\hline$E_{11}$ & $220 \mathrm{GPa}$ & $E_{22}$ & $7 \mathrm{GPa}$ & $E_{33}$ & $7 \mathrm{GPa}$ \\
$G_{23}$ & $2.9 \mathrm{GPa}$ & $G_{31}$ & $5 \mathrm{GPa}$ & $G_{12}$ & $5 \mathrm{GPa}$ \\
$\nu_{23}$ & 0.2 & $\nu_{31}$ & 0.35 & $\nu_{12}$ & 0.35 \\
$\rho$ & $1,600 \mathrm{~kg} / \mathrm{m}^{3}$ & & & & \\
$X_{t}$ & $1,100 \mathrm{MPa}$ & $Y_{t}$ & $50 \mathrm{MPa}$ & $Z_{t}$ & $50 \mathrm{MPa}$ \\
$X_{c}$ & $1,100 \mathrm{MPa}$ & $Y_{c}$ & $150 \mathrm{MPa}$ & $Z_{c}$ & $150 \mathrm{MPa}$ \\
$S_{y z}$ & $75 \mathrm{MPa}$ & $S_{z x}$ & $75 \mathrm{MPa}$ & $S_{x y}$ & $75 \mathrm{MPa}$ \\
\hline
\end{tabular}

\section{References}

ABAQUS (2008) Abaqus theory manual, v6.8 edn. Dassault Systèmes Simulia Corp., Providence

Abdalla MM, Setoodeh S, Gürdal Z (2007) Design of variable stiffness composite panels for maximum fundamental frequency using lamination parameters. Compos Struct 81(2):283-291

Adali S, Lene F, Duvaut G, Chiaruttini V (2003) Optimization of laminated composites subject to uncertain buckling loads. Compos Struct 62:261-269

Adams DB, Watson LT, Gürdal Z, Anderson-Cook CM (2004) Genetic algorithm optimization and blending of composite laminates by locally reducing laminate thickness. Adv Eng Softw 35:34-43

Akbulut M, Sonmez FO (2008) Optimum design of composite laminates for minimum thickness. Comput Struct 86(21-22):19741982. doi:10.1016/j.compstruc.2008.05.003

Autio M (2000) Determining the real lay-up of a laminate corresponding to optimal lamination parameters by genetic search. Struct Multidisc Optim 20:301-310

Aymerich F, Serra M (2008) Optimization of laminate stacking sequence for maximum buckling load using the ant colony optimization (aco) metaheuristic. Compos, Part A 39:262-272

Beluch W (2007) Evolutionary identification and optimization of composite structures. Mech Adv Mater Struct 14(8):677-686

Ben-Tal A, Kočvara M, Nemirovski A, Zowe J (1999) Free material design via semidefinite programming: the multiload case with contact conditions. SIAM J Optim 9(4):813-832

Bentley P (ed) (1999) Evolutionary design by computers. Morgan Kaufmann, San Francisco

Bertsekas DP (1982) Constrained optimization and lagrange multiplier methods. Academic

Bruyneel M, Fleury C (2002) Composite structures optimization using sequential convex programming. Adv Eng Softw 33(7-10):697711. doi:10.1016/S0965-9978(02)00053-4

Cantu-Paz E (1997) Designing efficient master-slave parallel genetic algorithms. Tech. rep., University of Illinois at UrbanaChampaign, Illinois Genetic Algorithms Laboratory, Urbana, IL. IIIGAL Report 97004

Cho H, Rowlands R (2007) Reducing tensile stress concentration in perforated hybrid laminate by genetic algorithm. Compos Sci Technol 67(13):2877-2883. doi:10.1016/j.compscitech.2006. 09.022

Duvaut G, Terrel G, Léné F, Verijenko VE (2000) Optimization of fiber reinforced composites. Compos Struct 48(1-3):83-89. doi:10.1016/S0263-8223(99)00077-X

Farshi B, Rabiei R (2007) Optimum design of composite laminates for frequency constraints. Compos Struct 81:587-597

Foldager J, Hansen JS, Olhoff N (1998) A general approach forcing convexity of ply angle optimization in composite laminates. Struct Multidisc Optim 16:201-211. doi:10.1007/BF01202831

Fukunaga H, Vanderplaats GN (1991) Strength optimization of laminated composites with respect to layer thickness and/or layer orientation angle. Comput Struct 40:1429-1439

Gen M, Cheng R (2000) Genetic algorithms and engineering optimization. Wiley, New York

Giger M (2007) Representation concepts in evolutionary algorithmbased structural optimization. PhD thesis, Swiss Federal Institute of Technology ETH, Zürich, diss. ETH no. 17017

Giger M, Ermanni P (2005) Developement of CFRP racing motorcycle rims using a heuristic evolutionary algorithm approach. Struct Multidisc Optim 30(1):54-65. doi:10.1007/s00158-004-0471-3

Giger M, Keller D, Ermanni P (2008) A graph-based parameterization concept for global laminate optimization. Struct Multidisc Optim 36(3):289-305 
Grenestedt J (1990) Composite plate optimization only requires one parameter. Struct Optim 2:29-37

Grenestedt J (1991) Layup optimization against buckling of shear panels. Struct Optim 3:115-120

Gürdal Z, Haftka RT, Hajela P (1999) Design and optimization of laminated composite materials. Wiley

Hammer VB, Bendsøe MP, Lipton R, Pedersen P (1997) Parameterization in laminate design for optimial compliance. Int J Solids Struct 34:415-434

Herencia JE, Weaver PM, Friswell MI (2007) Optimization of long anisotropic laminated fiber composite panels with t-shaped stiffeners. AIAA J 45(10):2497-2509

Hörnlein HREM, Kočvara M, Werner R (2001) Moped: an integrated designer tool for material optimization. Tech. rep. 279, Universität Erlangen, Institut für angewandte Mathematik

Huang J, Haftka R (2005) Optimization of fiber orientations near a hole for increased load-carrying capacity of composite laminates. Struct Multidisc Optim 30:335-341. doi:10.1007/s00158005-0519-z

Johansen LS, Lund E, Kleist J (2009) Failure optimization of geometrically linear/nonlinear laminated composite structures using a twostep hierarchical model adaptivity. Comput Methods Appl Mech Eng 198(30-32):2421-2438. doi:10.1016/j.cma.2009.02.033

Kaufmann M (2006) Zonal graph-based laminate optimization. Master's thesis, ETHZ

Keller D (2010a) Evolutionary design of laminated composite structures. PhD thesis, ETH Zürich, ETH diss. no. 19011

Keller D (2010b) A local search embedded in the variation state of an evolutionary algorithm. Adv Eng Softw, submitted

Khosravi P, Sedaghati R (2008) Design of laminated composite structures for optimum fiber direction and layer thickness, using optimality criteria. Struct Multidisc Optim 36(2):159-167. doi:10.1007/s00158-007-0207-2

Kicinger R, Arciszewski T, Jong KD (2005) Evolutionary computation and structural design: a survey of the state-of-the-art. Comput Struct 83(23-24):1943-1978

Kim JS (2007) Development of a user-friendly expert system for composite laminate design. Compos Struct 97:76-83

Kočvara M, Zowe J (1998) Free material optimization. In: Documenta mathematica. Journal der deutschen Mathematikervereinigung. Proceedings of the international congress of mathematicians. Deutsche Mathematikervereinigung, vol 3, pp 707-716

Kogiso N, Watson LT, Gürdal Z, Haftka RT (1994) Genetics algorithms with local improvement for composite laminte design. Struct Optim 7(4):207-218

König O (2004) Evolutionary design optimization: tools and applications. PhD thesis, Swiss Federal Institute of Technology, Zürich, diss. ETH no. 15486

Le Riche R, Haftka RT (1995) Improved genetic algorithm for minimum thickness composite laminate design. Compos Eng 5:143161

Le Riche RG, Knopf-Lenoir C, Haftka RT (1995) A segregated genetic algorithm for constrained structural optimization. In: Eshelman L (ed) Proceedings of the sixth international conference on genetic algorithms. Morgan Kaufmann, San Francisco, pp 558-565

Ledermann C (2006) Parametric associative CAE methods in preliminary aircraft design. PhD thesis, Swiss Federal Institute of Technology, Zürich, diss. ETH no. 16778

Liu B, Haftka R, Akgun M, Todoroki A (2000) Permutation genetic algorithm for stacking sequence design of composite laminates. Comput Methods Appl Mech Eng 186:357-372

Lopez R, Luersen M, Cursi E (2009) Optimization of laminated composites considering different failure criteria. Compos, Part B 40:731-740
Lund E (1994) Finite element based design sensitivity analysis and optimization. $\mathrm{PhD}$ thesis, Institute of Mechanical Engineering, Aalborg University

Lund E, Stegmann J (2005) On structural optimization of composite shell structures using a discrete constitutive parametrization. Wind Energy 8(1):109-124. doi:10.1002/we.132

Lund E, Stegmann J (2006) Eigenfrequency and buckling optimization of laminated composite shell structures using discrete material optimization. Solid Mech Appl 3:147-156

Mateus HC, Soares CMM, Soares CAM (1991) Sensitivity analysis and optimal design of thin laminated composite structures. Comput Struct 41:501-508

Mateus H, Rodrlgues H, Soares CM, Soares CM (1997) Sensitivity analysis and optimization of thin laminated structures with a nonsmooth eigenvalue based criterion. Struct Optim 14:219 224

Matsuzaki R, Todoroki A (2007) Stacking-sequence optimization using fractal branch-and-bound method for unsymmetrical laminates. Compos Struct 78:537-550

Miki M (1982) Material design of composite laminates with required in-plane elastic properties. In: Hayashi T, Kawata K, Umekawa M (eds) Progress in science and engineering of composites. Tokyo, ICCM-IV, pp 1725-1731

Moita J, Barbosa JI, Soares CM, Soares CM (2000) Sensitivity analysis and optimal design of geometrically non-linear laminated plates and shells. Comput Struct 76:407-420

Naik GN, Gopalakrishnan S, Ganguli R (2008) Design optimization of composites using genetic algorithms and failure mechanism based failure criterion. Compos Struct 83(4):354-367. doi:10.1016/j.compstruct.2007.05.005

Omkar S, Mudigere D, Naik GN, Gopalakrishnan S (2008) Vector evaluated particle swarm optimization (VEPSO) for multi-objective design optimization of composite structures. Comput Struct 86(1-2):1-14. doi:10.1016/j.compstruc.2007. 06.004

Pai N, Kaw A, Weng M (2003) Optimization of laminate stacking sequence for failure load maximization using tabu search. Compos, Part B 43:405-413

Park CH, Lee WI, Han WS, Vautrin A (2008) Improved genetic algorithm for multidisciplinary optimization of composite laminates. Comput Struct 86:1894-1903

Pedersen P (1989) On optimal orientation of orthotropic materials. Struct Multidisc Optim 1:101-106. doi:10.1007/BF0163766610. 1007/BF01637666

Pedersen P (1991) On thickness and orientational design with orthotropic materials. Struct Multidisc Optim 3(2):69-78

Pelegri AA, Kedlaya DN (2008) Design of composites using a generic unit cell model coupled with a hybrid genetic algorithm. Compos, Part A 39:1433-1443

Pelletier JL, Vel SS (2006) Multi-objective optimization of fiber reinforced composite laminates for strength, stiffness and minimal mass. Comput Struct 84:2065-2080

Prager W (1970) Optimization of structural design. J Optim Theory Appl 6(1):1-21. doi:10.1007/BF00927037

Rao ARM, Arvind N (2005) A scatter search algorithm for stacking sequence optimisation of laminate composites. Compos Struct 70:383-402

Rastogi N (2004) Stress analysis and lay-up optimization of an allcomposite pick-up truck chassis structure. Tech. rep., Visteon Corporations

Roche D (2004) Formula 1 monocoque composite lay-up optimization. Tech. rep., P+Z Engineering Ltd

Sauter M (2008) A graph-based optimization method for the design of compliant mechanisms and structures. PhD thesis, ETH Zurich, diss. ETH no. 17787 
Seresta O, Gürdal Z, Adams DB, Watson LT (2007) Optimal design of composite wing structures with blended laminates. Compos, Part B 38:469-480,731

Soremekun GAE (1997) Genetic algorithms for composite laminate design and optimization. Master's thesis, Virginia Tech

Stegmann J (2005) Analysis and optimization of laminated composite shell structures. PhD thesis, Institute of Mechanical Engineering, Aalborg University, Pontoppidanstraede 101, DK-9220 Aalborg East, Denmark

Stegmann J, Lund E (2005) Discrete material optimization of general composite shell structures. Int J Numer Methods Eng 62(14): 2009-2027

Suresh S, Sujit P, Rao A (2007) Particle swarm optimization approach for multi-objective composite box-beam design. Compos Struct 81(4):598-605. doi:10.1016/j.compstruct.2006.10.008

Todoroki A, Haftka RT (1997) Stacking sequence matching by twostage genetic algorithm with consanguineous initial population. In: AIAA/ASME/ASCE/AHS/ASC structures, structural dynamics, and materials conference and exhibit

Todoroki A, Haftka R (1998) Stacking sequence optimization by a genetic algorithm with a new recessive gene like repair strategy. Compos, Part B: Eng 29:277-285

Todoroki A, Ishikawa T (2004) Design of experiments for stacking sequence optimizations with genetic algorithm using response surface approximation. Compos Struct 64:349-357

Todoroki A, Sekishiro M (2007) New iteration fractal branch and bound method for stacking sequence optimizations of multiple laminates. Compos Struct 81:419-426
Topal U, Uzman U (2008) Strength optimization of laminated composite plates. J Compos Mater 42:1731-1746. doi:10.1177/ 0021998308093368

Tsau LR, Liu CH (1995) A comparison between two optimization methods on the stacking sequence of fiber-reinforced composite laminate. Comput Struct 55:515-525. doi:10.1016/00457949(95)98877-S

van Keulen F, Haftka R, Kim N (2005) Review of options for structural design sensitivity analysis. Part 1: linear systems. Comput Methods Appl Mech Eng 194:3213-3243

Vannucci P, Barsotti R, Bennati S (2009) Exact optimal flexural design of laminates. Compos Struct 90(3):337-345. doi:10.1016/ j.compstruct.2009.03.017

Walker M, Smith RE (2003) A technique for the multiobjective optimisation of laminated composite structures using genetic algorithms and finite element analysis. Compos Struct 62:123-128

Wintermantel M (2004) Design-encoding for evolutionary algorithms in the field of structural optimization. PhD thesis, Swiss Federal Institute of Technology, Zürich, diss. ETH no. 15323

Zehnder N (2008) Global optimization of laminated structures. PhD thesis, ETH Zürich, diss. ETH no. 17573

Zehnder N, Ermanni P (2006) A methodology for the global optimization of laminated composite structures. Compos Struct 72: 311-320

Zowe J, Kočvara M, Bendsøe MP (1997) Free material optimization via mathematical programming. In: Liebling TM, de Werra D (eds) Mathematical programming, vol 79. Mathematical Programming Society, Elsevier, pp 445-466 